\title{
Mixed-integer formulations for the Capacitated Rank Pricing Problem with envy
}

\author{
Concepción Domínguez ${ }^{1,2,3} \quad$ Martine Labbé ${ }^{1,2} \quad$ Alfredo Marín ${ }^{3}$ \\ ${ }^{1}$ Université Libre de Bruxelles, Belgium \\ 2 Inria Lille-Nord Europe, France \\ ${ }^{3}$ Universidad de Murcia, Spain \\ December 13, 2021
}

\begin{abstract}
Pricing under a consumer choice model has been extensively studied in economics and revenue management. In this paper, we tackle a generalization of the Rank Pricing Problem (RPP), a multi-product pricing problem with unit-demand customers and a ranking-based consumer choice model. We generalize the RPP assuming that each product has a limited amount of copies for sale, and we call this extension the Capacitated Rank Pricing Problem (CRPP). We compare the envy-free allocation of the products (a fairness criterion requiring that customers receive their highest-ranked product given the pricing) with the envy version of the problem. Next, we focus on the CRPP with envy. We introduce two integer linear formulations for the CRPP and derive valid inequalities leveraging the structure of the problem. Afterwards, we develop separation procedures for the families of valid inequalities of greater size. The performance of the formulations and the resolution algorithms developed is tested by means of extensive computational experiments.
\end{abstract}

Keywords: Rank Pricing Problem, Ranking-based Consumer Models, Combinatorial Optimization, Integer Programming, Valid Inequality, Bilevel Programming

\section{Introduction}

Developing an adequate pricing strategy is a crucial decision faced daily by companies in retail and manufacturing. Furthermore, the recent availability of data regarding customers' purchasing behavior and the conviction that it constitutes a high impact factor in the overall profitability of firms have motivated the proliferation of pricing problems under consumer choice models.

There are many variations on product pricing models under a consumer choice model depending on the setting (for an overview, see Van Ryzin and Talluri [22]). An important line of research is pricing under the assumption of unit-demand customers (interested in purchasing at most one product) whose product choices are determined entirely by their reservation prices. In this context, each customer owns a reservation price for each product that reflects the maximum price the customer is willing to pay for the given product, commonly known as his willingness to pay. Then the customer selects the product that 
maximizes his utility or surplus, i.e. the difference between his reservation price and the price of the product. Some references in pricing within the utility-maximization framework include Dobson and Kalish [7, 8], Heilporn et al. [13], Fernandes et al. [9], Myklebust et al. [16] and Shioda et al. [19, 20].

Utility-based models assume that customers follow compensatory decision processes, where high levels on some attributes of a product (such as the price) can compensate for low levels on other aspects. However, customers sometimes follow less rational decision processes, such as heuristics or shortcuts, to speed up their decision. In these cases, they make non-compensatory decision processes (such as the lexicographic rule, where customers rate certain features of the products by importance and purchase accordingly). In fact, non-compensatory considerations tend to be more significant in important decisions, such as in residential location and vehicle type choices (Swait [21]). A ranking-based model can accommodate both compensatory and non-compensatory decision processes (if the prices are not considered as product attributes). In this work, we focus on the problem of pricing a product line assuming that customers' product selection is based on a ranking of the products.

Specifically, we study a generalization of the Rank Pricing Problem (RPP). The RPP is given by unit-demand customers, that is, customers interested in a subset of the products offered by the company and who intend to buy at most one of them. To do so, the customers rank a subset of the products of the company that they consider, making a ranked list. They are also endowed with a positive budget that is independent of the products they desire. Once the prices are established by the company, they will purchase their highest ranked one among the ones with a price below their budget (if any). The $\mathrm{RPP}$ is classified as a multi-product pricing optimization problem with a non-parametric ranking-based consumer choice model.

The RPP was introduced by Rusmevichientong et al. [17] and Rusmevichientong [18]. They leverage data collected through the Auto Choice Advisor (ACA) website to optimize the price of General Motors' vehicles. The ACA website creates a list of recommended vehicles based on the budget constraint of the consumer and his requirements. Rusmevichientong et al. introduce the RPP, prove that it is NP-complete in the strong sense and derive a heuristic approximation algorithm for the RPP (with performance bounds) under the assumption of a price-ladder constraint (a constraint on the ordering of prices). They apply their algorithm to a real data set from the ACA website, providing an analysis of the current pricing scheme at General Motors and suggesting improvements that might lead to a more effective one. Aggarwal et al. [1] study variants of the original models proposed in [17]. They show that the RPP with price-ladder can be reduced to the Max Pricing, a problem of profit maximization proposed in [17] where customers purchase the most expensive product under their budget. They also derive a polynomial time approximation scheme for the RPP with price-ladder. Briest and Krysta [4] prove that the previous algorithm is essentially best possible, and prove near-tight hardness results for some versions of the RPP (including the most general). Calvete et al. [5] introduce the first integer linear formulations for the RPP, and Domínguez et al. [6] present integer linear formulations and results for the Rank Pricing Problem with Ties (RPPT), a variant of the RPP that allows for ties in the ranking of the products.

In this paper, we tackle an extension of the RPP. In the first place, we assume that 
each customer has a different reservation price that reflects his willingness to pay for each product of his interest, and therefore he purchases the highest-ranked product among the ones priced under their corresponding reservation prices. Furthermore, we assume that the company can only offer a limited amount of copies of each product for sale and might not have enough supply to satisfy its clients. We have named this problem the Capacitated Rank Pricing Problem (CRPP). One application of the CRPP consists in pricing tickets for concerts or other types of events. In this case, different seats have different prices that are related to their characteristics (distance to the event, visibility, etc.). Some customers prefer to pay a lower price for a worse seat but, if those tickets are sold out, they might be willing to pay more for a better one so as not to miss the event. Other customers actually prefer the best tickets available. Hence, it fits the capacity restriction and the customers' representation through a list of preferences and reservation prices well.

Limiting the number of copies of each product implies that in some solutions, some customers are unable to purchase their favorite product because it has sold out, even if they can afford it. This results in two versions of the problem that differ in the type of solution sought by the company. On the one hand, the company may opt for a solution in which its profit is maximized restricting the search to the solutions that avoid (possible) conflicts among customers. In this case, it shall choose what is known as an envy-free solution, that is, a solution in which it can provide to each customer the product he prefers the most among all those that have a price lower than his reservation price. An envy-free solution always exists, it suffices to consider the solution where the products are very expensive and no customer can afford them, so there is no possible envy among customers. Guruswami et al. 12 introduce the profit maximization problem assuming that customers maximize their utility and are envy-free for both unit-demand and singleminded customers. They present an algorithm with logarithmic approximation guarantee for the envy-free unit-demand problem and prove APX-hardness. On the other hand, the company may choose to maximize its profit regardless of possible conflicts among customers. Such conflicts arise when a customer prefers a product more than the one he is given and he can afford it, but the product is not available because all the copies have been sold to other customers. In some settings, envy solutions can lead to dissatisfied customers, what can result in the loss of clients in the long term.

Envy and envy-free allocations have different characteristics. Both allocations can be considered equitable, in the sense that the prices are settled according to the preferences of the customers and their reservation prices, and there is a unique price for each product. However, although an envy-free pricing (i.e. a pricing that admits a feasible, envy-free allocation of products) prevents the customers' possible displeasure with their purchase, it generally provides a smaller profit for the company than a solution that allows for envy. Moreover, the envy setting also assumes that customers might come across products they are willing to buy and they can afford but are sold out, which is a realistic assumption in the context of a limited supply of products. Lastly, like in the RPP, if the pricing is envy-free the allocation of the products to the customers is uniquely determined. Thus, any envy-free pricing leads to a unique feasible allocation of the products. On the other hand, if the envy-freeness is not required, a pricing can lead to multiple feasible allocations of the products to the customers that yield different profit. As a consequence, a feasible solution in the envy-free setting is also a solution for the envy setting, and moreover the version of the CRPP that allows for envy is inherently more difficult than the envy-free 
one.

To the best of our knowledge, the CRPP has not yet been studied in the literature. We address the resolution of the envy version of the CRPP by means of discrete optimization methods. Since the only formulations and exact resolution algorithms proposed for variants of the RPP were developed in [5] and [6], our results are built on the ones designed in these two papers. However, the fact that we allow for envy in the CRPP makes this version of the problem intrinsically different from the previous ones. To begin with, the assignment of the products to the customers (given a fixed pricing) is straightforward in the envy-free case. However, in this work we show that the assignment problem associated to the CRPP with envy is NP-complete. Furthermore, widely used methods such as Benders decomposition, used in [6] as a resolution method for a variant of the RPP, do not apply to this version of the problem because it is not possible to relax the integrality constraints of the decision purchase variables. Other procedures like the preprocessing techniques developed in [5] and [6] do not apply either in this case due to the envy.

We introduce two integer linear formulations for the CRPP, one with three-index customer decision variables and a second one that makes use of a much smaller set of variables but generally provides worse linear relaxation bounds. These formulations have similarities with the ones proposed in [5] and [6], but the constraints related to the preferences of the customers differ and the capacity is modeled introducing a new set of variables. The three-index formulation is strengthened by means of alternative sets of constraints derived leveraging the capacity constraints and the three-index variables. These families are based on the limited supply assumption, so they do not apply to the uncapacitated versions of the problem. As for the reduced formulation, three families of valid inequalities of exponential size are presented along with their respective separation procedures to include them dynamically in branch-and-cut frameworks. One of these families was proposed for the RPP in [5] as part of a family of exponential size. Here we prove that the family that we derive in this paper (which is polynomial in size) dominates the previous one. Finally, we compare the performance of both models and the different resolution algorithms described by means of extensive computational experiments.

The rest of the paper is organized as follows. In Section 2, we introduce the notation, explain the difference between the envy-free and the envy case by means of an example, and prove that the assignment of the CRPP with envy is NP-complete. In Section 3, we introduce the three-index integer linear formulation and several families of valid inequalities derived to strengthen it. Section 4 is devoted to the presentation of the reduced formulation. In Section 5 we include the families of valid inequalities for the reduced model and the separation procedures developed to incorporate constraints in a branchand-cut fashion. Section 6 includes the computational study, and the conclusions are provided in Section 7 .

\section{Notation and problem description}

Recall that the CRPP aims at maximizing the profit of a company selling different products with a limited supply, taking into account the preferences and reservation prices of the unit-demand customers. Since we tackle the version of the problem that allows for 
envy, once the prices are settled, clients are assigned their highest-ranked product among the ones that are not sold out and have a price below the corresponding reservation price (if any).

Let us define $K=\{1, \ldots,|K|\}$ as the set of customers and $I=\{1, \ldots,|I|\}$ as the set of products, with $c_{i}$ the number of copies for sale of product $i, \forall i \in I$. Each customer $k \in K$ has a subset of products $I^{k} \subseteq I$ he could potentially purchase, and he ranks the products in $I^{k}$ from the best to the worst (ties in the ranking are not allowed). If $k$ ranks product $i$ higher than product $j$, we say that $k$ prefers $i$ to $j$, and we denote $i \prec_{k} j$. Furthermore, $\left\{j \in I^{k}: j \preceq_{k} i\right\}=\{i\} \cup\left\{j \in I^{k}: j \prec_{k} i\right\}$. For a given product $i \in I$, we define $K_{i}:=\left\{k \in K: i \in I^{k}\right\}$ as the set of customers that could purchase product $i \in I$. Without loss of generality, we assume $I^{k} \neq \emptyset \forall k \in K, K_{i} \neq \emptyset \forall i \in I$.

Each customer $k$ has a reservation price for each product $i \in I^{k}$. Since different customers may have the same reservation price for the same product $i$, we define set $M_{i}=\left\{1, \ldots,\left|M_{i}\right|\right\}$ as the set of indices that refer to the different reservation prices of the customers, and $\left(b_{i}^{m}\right)_{m \in M_{i}}$ as the set of different reservation prices for $i$, so that $b_{i}^{m_{1}}<b_{i}^{m_{2}}$ if $m_{1}<m_{2}$. To represent the reservation prices of a customer for a product, we define a function $\sigma_{i}: K_{i} \rightarrow M_{i} \forall i \in I$ such that $\sigma_{i}(k)=m$ if the reservation price of $k$ is the $m$-th smallest reservation price $b_{i}^{m}$.

In Rusmevichientong et al. [17] it is proved that there always exists an optimal solution of the RPP in which the price of any product $i$ is equal to the budget of a customer. This result also applies to the CRPP with different reservation prices for each product, instead of a positive budget for all of them. With the aim of contradiction, consider an optimal pricing vector and allocation of products with a product $i$ of price $p_{i}$, with $b_{i}^{m}<p_{i}<b_{i}^{m+1}$ (and assume there is at least one copy of $i$ sold). Then setting the price of $i$ to $b_{i}^{m+1}$ (and keeping the prices of the rest of the products unaltered), we obtain a new pricing vector for which the previous allocation of products is also feasible, because the subset of products under the reservation price of any customer remain the same. However, for any customer purchasing $i$, the company is obtaining $b_{i}^{m+1}$ instead of $p_{i}$, so the new pricing vector yields greater profit, which is a contradiction. Finally, for $k \in K_{i}, M_{i}^{k}:=\left\{m \in M_{i}: m \leq \sigma_{i}(k)\right\}$ represents the set of indices $m$ of candidate prices $b_{i}^{m}$ at which $k$ could purchase $i$ in a feasible solution.

As explained in the introduction, the feasible solutions of the CRPP differ depending on the setting that we choose. If we solve the envy-free version of the CRPP, then for a fixed pricing there is a unique feasible allocation of the products to the customers because each of them purchases the highest ranked product below his reservation price (if any). On the other hand, if we allow for envy in the solutions, different allocations with different revenues may exist. In the following, we illustrate the difference between an envy-free solution of the CRPP and a solution that allows for envy by means of the example of Figure 1. 


\begin{tabular}{|c|c|c|c|c|c|}
\hline & $\begin{array}{c}\text { Ticket } 1 \\
\text { (stalls or arena) }\end{array}$ & $\begin{array}{c}\text { Ticket } 2 \\
\left(1^{\text {st }} \text { Balcony }\right)\end{array}$ & & $\begin{array}{c}\text { Ticket } 1 \\
\text { (stalls or arena) }\end{array}$ & $\begin{array}{c}\text { Ticket } 2 \\
\left(1^{\text {st }} \text { Balcony }\right)\end{array}$ \\
\hline & $c_{1}=2$ & $c_{2}=2$ & & $c_{1}=2$ & $c_{2}=2$ \\
\hline Cust. 1 & $2 \mid 50$ & $1 * \mid 30$ & Cust. 1 & $2^{*} \mid 50$ & $1 \mid 30$ \\
\hline Cust. 2 & $1^{*} \mid 40$ & $2 \mid 40$ & Cust. 2 & $1 \mid 40$ & $2^{*} \mid 40$ \\
\hline Cust. 3 & $2^{*} \mid 30$ & $1 \mid 20$ & Cust. 3 & $2^{*} \mid 30$ & $1 \mid 20$ \\
\hline Opt. price & 30 & 30 & Opt. price & 30 & 40 \\
\hline
\end{tabular}

(a) Envy-free solution

(b) Solution that allows for envy

Figure 1: Optimal solutions of an instance of the CRPP

Example 2.1. Figure 1 a shows an instance of the CRPP problem where different types of theater tickets for a specific event are sold to customers. In the example, there are $|K|=3$ customers and $|I|=2$ types of tickets. Ticket 1 is a ticket to see the performance from the stalls or arena sitting area, and Ticket 2 corresponds with a ticket in the $1^{\text {st }}$ Balcony of the theater. The number of copies $c_{i}$ of each type of ticket $i$ is represented right below the product. In our instance, there are two copies of each product/ticket for sale. The left number in an entry represents the preference of the customer for that product. If product $i$ is the highest ranked product for customer $k$, then the number represented is 1 ; and for his second product, the number is 2. The right number in each entry corresponds to the reservation price of that customer for that product. In this example, customers 1,2 and 3 have reservation prices of 30, 40 and 20 for product 2, respectively. Therefore, the number of different reservation prices for product 2 is $\left|M_{2}\right|=3$. As for the ordered reservation prices, they are $b_{2}^{1}=20, b_{2}^{2}=30$ and $b_{3}^{2}=40$. Lastly, the reservation prices for product 2 are related to the customers by means of function $\sigma_{2}$. For instance for customer 1 it holds $\sigma_{2}(1)=2$ because he owns the second lowest reservation price for product 2 . Similarly, $\sigma_{2}(2)=3$ and $\sigma_{2}(3)=1$. The optimal envy-free solution is obtained setting the prices depicted at the bottom of the table. Customers purchase the product whose preference is marked with an asterisk in the preference matrix, and the total revenue of the company is equal to 90 .

However, the revenue of the company increases if we allow for envy among customers. Figure $1 b$ contains the same instance with the optimal solution in the envy setting. In this solution, customer 2 is allocated product 2 even though product 1 is under his reservation price and he prefers 1 to 2 , because product 1 is sold out. Thus, this allocation of products is not feasible for the envy-free setting. The optimal value in this case is equal to 100. A different allocation of products (with the same pricing and with envy) that yields a smaller optimal value consists in assigning product 1 to customers 1 and 2. Product 2 remains unsold because customer 2 is purchasing product 1 and customers 1 and 3 have a reservation price below 40. In this case, the revenue is equal to 60.

We have just seen that, given a pricing, different allocations (in the envy setting) may have different revenue. This leads to the question of whether there exists an efficient 
algorithm for the problem of finding an allocation of maximum revenue in the restricted version of the CRPP where the prices are fixed. In the following subsection, we show that this problem is in fact NP-complete.

\subsection{Complexity of the non envy-free assignment problem of the CRPP}

In Rusmevichientong et al. [17] it is proved that the RPP is NP-complete in the strong sense, and hence so is the CRPP. We now consider a fixed pricing of the products in the CRPP, and study the assignment of the products to the customers. It is clear that in the envy-free case, the assignment of the products is straightforward: each customer purchases the highest ranked product in his list below his reservation price (if any). In this subsection, however, we prove that the non envy-free assignment of the CRPP is NP-complete. To do so, we consider a restricted case of the CRPP where all the prices of the products are fixed to one single price $p$ and the number of copies of each product is equal to one. We begin the subsection introducing a matching problem called the Stable Marriage problem with Ties and Incomplete Lists (SMTI). We then reduce an NP-hard particular case of the SMTI to our problem to prove the result. A general overview on algorithms for matching problems under preferences can be found in Manlove [14].

An instance of SMTI involves a set $U=\left\{u_{1}, \ldots, u_{n_{2}}\right\}$ of men, a set $W=\left\{w_{1}, \ldots, w_{n_{1}}\right\}$ of women, and a set $E \subseteq U \times W$ of acceptable man-woman pairs. Thus, each man $u_{i} \in U$ has an acceptable set of women $A\left(u_{i}\right)=\left\{w_{j} \in W:\left(u_{i}, w_{j}\right) \in E\right\}$ and likewise, each woman $w_{j}$ has an acceptable set of men $A\left(w_{j}\right)=\left\{u_{i} \in W:\left(u_{i}, w_{j}\right) \in E\right\}$. The agents are the men and women in $U \cup W$. Each agent $a_{k} \in U \cup W$ has a preference list in which he/she ranks $A\left(a_{k}\right)$. However, agents are allowed to express indifference in their preference lists. We denote $w_{j} \prec_{u_{i}} w_{j^{\prime}}$ when man $u_{i}$ prefers woman $w_{j}$ to $w_{j^{\prime}}$, and we use $w_{j} \sim_{u_{i}} w_{j^{\prime}}$ if $u_{i}$ is indifferent between two women $w_{j}$ and $w_{j^{\prime}}$. Specifically, $\sim_{k}$ is an equivalence relation (reflexive, symmetric, transitive) and there exists a linear order over the equivalence classes of $A\left(a_{k}\right)$ for each $k \in K$. The special case of SMTI in which every man-woman pair is acceptable and all the preferences are strict is called the Stable Marriage problem (SM). The SM was first studied by Gale and Shapley [11].

An assignment $M$ is a subset of $E$. If $\left(u_{i}, w_{j}\right) \in E$, then $u_{i}$ is assigned to $w_{j}$ and $w_{j}$ is assigned to $u_{i}$. For each $a_{k} \in U \cup W$, the set of assignees of $a_{k}$ in $M$ is denoted by $M\left(a_{k}\right)$. If $a_{k} \in U \cup W$ and $M\left(a_{k}\right)=\emptyset, a_{i}$ is unassigned; otherwise $a_{k}$ is assigned. A matching $M$ is an assignment such that $\left|M\left(a_{k}\right)\right| \leq 1 \forall a_{k}$.

Given a matching $M$ in an instance $I$ of SMTI, a pair $\left(u_{i}, w_{j}\right)$ is said to block $M$, or to be a blocking pair of $M$, if the following conditions are satisfied:

1. $u_{i}$ is unassigned or prefers $w_{j}$ to his assigned woman in $M$, and

2. $w_{j}$ is unassigned or prefers $u_{i}$ to her assigned man in $M$.

$M$ is said to be weakly stable (or simply stable) if it has no blocking pair. Every instance of SMTI has a stable matching, and finding one can be done in linear time. Furthermore, all the stable matchings in an instance of the SM have the same size. However, instances 
of the SMTI can have stable matchings of different sizes. In fact, the problem of finding a maximum cardinality weak stable matching in an instance of SMTI, called MAX-SMTI, is NP-hard. The NP-hardness holds even in the restricted case where the ties occur in the women's preference lists only, any tie forms the whole list in which it appears, and each tie is of length 2 (Manlove et al. [15, Theorem 2]). In the following, we reduce the restricted case of MAX-SMTI aforementioned to our problem to prove that the non envy-free assignment of the CRPP is NP-hard. Define the following decision problem:

Name: Non envy-free assignment of the CRPP

Instance: $K$ customers, $I$ products with the same fixed price, a number of copies of each product, a subset of acceptable products $I^{k}$ for each customer $k$, a preference list (with no ties) of products for each customer, integer reservation prices of the customers, an integer $T \in \mathbb{Z}^{+}$.

Question: Does the given instance admit a feasible non envy-free assignment of products of revenue equal to $T$ ?

Proposition 2.2. The non envy-free assignment of the CRPP is NP-complete, even if there is only one copy of each product and the price of every product is fixed to a common price 1.

Proof. It is easy to see that the non envy-free assignment of the CRPP is in NP. Given a pricing and an assignment of the products, checking for feasibility translates to checking that, for a given customer, all the products he prefers to the one he is assigned either are sold out or have a price above his reservation price. Clearly, this can be done in polynomial time. To show NP-hardness, we reduce the particular version of MAX-SMTI where ties occur in the women's preference lists only, any tie forms the whole list in which it appears, and each tie is of length 2 .

Let $I$ be an instance of MAX-SMTI, $U=\left\{u_{1}, \ldots, u_{n_{2}}\right\}$ the set of men, $W=\left\{w_{1}, \ldots, w_{n_{1}}\right\}$ the set of women, and $E \subseteq U \times W$. Assume that the preference list of every man has no ties, and that every woman $w_{j}$ has an acceptable set $A\left(w_{j}\right)$ with two men and is indifferent between them. We construct an instance $I^{\prime}$ of the CRPP as follows: let $U$ be the set of customers, and $W$ be the set of products. The acceptable products for a customer coincide with the acceptable women for the corresponding man and his preference list is also the same. The set of customers interested in a product $w_{j}$ is defined as the set $A\left(w_{j}\right)$. Assume that there is only one copy of each product $w_{j}$. Set the price of every product to 1. Finally, for each customer $u_{i}$, set the reservation price of $w_{j}$ equal to 0 if $w_{j} \notin A\left(u_{i}\right)$, and equal to 1 otherwise. In this way, every customer can afford every product in his list of preference. We claim that the SMTI instance $I$ has a (weak) stable matching of size $T$ if and only if $I^{\prime}$ admits a feasible solution of revenue $T$.

For, suppose that $I$ has a stable matching $M$ of size $T$. We construct an assignment of products $M^{\prime}$ to customers assigning product $w_{j}$ to customer $u_{i}$ for each $\left(u_{i}, w_{j}\right) \in M$. Clearly, the revenue of this assignment is equal to the number of customers that purchase, i.e. T. Now suppose, with the aim of contradiction, that the assignment is not feasible. Then there exists a customer $u_{i}$ that has been assigned product $w_{j}$ but he prefers product $w_{j^{\prime}}$. Furthermore, $u_{i} \in A\left(w_{j^{\prime}}\right)$ and $w_{j^{\prime}}$ is not sold out. But then $\left(u_{i}, w_{j^{\prime}}\right)$ is a blocking pair in $M$, since $u_{i}$ prefers $w_{j^{\prime}}$ to $w_{j}$ and $w_{j^{\prime}}$ is unassigned.

Conversely, suppose that $M^{\prime}$ is a feasible assignment of the products to the customers 
with revenue $T$. Then, $M=\left\{\left(u_{i}, w_{j}\right) \in E: u_{i}\right.$ purchases product $w_{j}$ in $\left.M^{\prime}\right\}$ is a stable matching of size $T$ of $I$ of SMTI. Otherwise, let $\left(u_{i}, w_{j^{\prime}}\right)$ block $M$. Then $w_{j^{\prime}}$ is unassigned (because every men is tied in the list of preferences of $w_{j^{\prime}}$ ), and there exists $w_{j}$ such that $\left(u_{i}, w_{j}\right) \in E$ and $u_{i}$ prefers $w_{j^{\prime}}$ to $w_{j}$. But then the assignment $M^{\prime}$ is not feasible in the CRPP, because $u_{i}$ is purchasing $w_{j}$ but he prefers $w_{j^{\prime}}$, and $w_{j^{\prime}}$ is not sold out.

Having seen that the assignment of products is intrinsically different in the envy and envy-free versions of the CRPP, we now provide the formulations and results for the envy version of the problem.

\section{Three-index mixed integer formulation for the CRPP}

We first define the variables required to introduce our three-index formulation for the CRPP named (3ICM). We define binary variable $v_{i}^{m}, \forall i \in I, \forall m \in M_{i}$, that takes value 1 if the price of product $i$ is equal to the $m$-th smallest reservation price $b_{i}^{m}$. Next, to represent the purchasing decision of a customer, we define binary variable $y_{i}^{k m}, \forall k \in K$, $i \in I^{k}, m \in M_{i}^{k}$, that takes value 1 if customer $k$ purchases product $i$ at price $b_{i}^{m}$. Lastly, we define binary variable $u_{i}^{m}, \forall i \in I, m \in M_{i}$, which takes value 1 if that product $i$ sells out at price $b_{i}^{m}$. Formulation (3ICM) is as follows:

$$
\begin{aligned}
\text { (3ICM) } \max _{v, y, u} & \sum_{k \in K} \sum_{i \in I^{k}} \sum_{m \in M_{i}^{k}} b_{i}^{m} y_{i}^{k m} \\
\text { s.t. } & \sum_{i \in I^{k}} \sum_{m \in M_{i}^{k}} y_{i}^{k m} \leq 1 \quad \forall k \in K, \\
& \sum_{m \in M_{i}} v_{i}^{m} \leq 1 \quad \forall i \in I, \\
& \sum_{k \in K_{i}} \sum_{m \in M_{i}^{k}} y_{i}^{k m} \leq c_{i} \quad \forall i \in I, \\
& y_{i}^{k m} \leq v_{i}^{m} \quad \forall k \in K, i \in I^{k}, m \in M_{i}^{k}, \\
& u_{i}^{m} \leq v_{i}^{m} \quad \forall i \in I, m \in M_{i}, \\
& c_{i} \sum_{m \in M_{i}} u_{i}^{m} \leq \sum_{k \in K_{i}} \sum_{m \in M_{i}^{k}} y_{i}^{k m} \quad \forall i \in I, \\
& \sum_{m \in M_{i}} u_{i}^{m}+\left(c_{i}-1\right) \sum_{m \in M_{i}} v_{i}^{m} \geq \sum_{k \in K_{i}} \sum_{m \in M_{i}^{k}} y_{i}^{k m} \quad \forall i \in I, \\
& \sum_{m \in M_{i}^{k}} v_{i}^{m} \leq \sum_{m \in M_{i}^{k}} u_{i}^{m}+\sum_{\substack{j \in I \\
j \leq k^{i}}} \sum_{m \in M_{j}^{k}} y_{j}^{k m} \quad \forall k \in K, i \in I^{k}, \\
& v_{i}^{m}, u_{i}^{m} \in\{0,1\} \quad \forall i \in I, m \in M_{i}, \\
& y_{i}^{k m} \in\{0,1\} \quad \forall k \in K, i \in I^{k}, m \in M_{i}^{k} .
\end{aligned}
$$

Constraints (1b) ensure that customers are unit-demand. Constraints (1c) guarantee that each product has at most one price. If $\sum_{m \in M_{i}} v_{i}^{m}=0$, then the product is not proposed to the customers (or equivalently its price is set arbitrarily high). Constraints 
(1d) are the capacity constraints, and they assure the capacity is not exceeded. Constraints (1e) guarantee that customer $k$ purchases product $i$ at its right price. Constraints (1f) $-(1 \mathrm{~h})$ force the $u$-variables to take their right value. Constraints (1fi) establish that a product $i$ can only sell out at price $b_{i}^{m}$ (i.e. $u_{i}^{m}=1$ ) if it has such price. Constraints $(1 \mathrm{~g})$ force $u_{i}^{m}$ to be 0 when $\sum_{k \in K_{i}} \sum_{m \in M_{i}^{k}} y_{i}^{k m}<c_{i}$, and (1h) imply $u_{i}^{m}=1$ when $\sum_{k \in K_{i}} \sum_{m \in M_{i}^{k}} y_{i}^{k m}=c_{i}$. Constraints (1i) are the preference constraints and guarantee that the preferences are satisfied when the products are not sold out. When $\sum_{m \in M_{i}^{k}} v_{i}^{m}=1$, customer $k$ can afford product $i$. Therefore, either $\sum_{m \in M_{i}^{k}} u_{i}^{m}=1$, that is, the product is sold out, or customer $k$ must purchase $i$ or a product he prefers over $i$, so $\sum_{\substack{j \in I: \\ j \leq k^{i}}} \sum_{m \in M_{j}^{k}} y_{j}^{k m}=1$. Finally, the objective function $1 \mathrm{a}$ is the revenue of the company.

The set of $u$-variables used to derive formulation (3ICM) is not essential, in the sense that a formulation can be derived using only the sets of $v$-and $y$-variables. However, in the following we introduce several sets of valid inequalities that can strengthen formulation (3ICM), some of which incorporate $u$-variables, so this is the motivation to include them. First, we give a necessary definition:

Definition 3.1. Let $i$ be a product and $c_{i}$ the number of copies available. Then we define $c_{i}^{m}$ as the minimum between the capacity $c_{i}$ and the number of customers that can purchase $i$ in a feasible solution at price $b_{i}^{m}$, i.e. $c_{i}^{m}:=\min \left\{c_{i},\left|\left\{k \in K_{i}: \sigma_{i}(k) \geq m\right\}\right|\right\}$.

This definition allows for the introduction of a strengthened set of capacity constraints:

Proposition 3.2. The set of constraints

$$
\sum_{k \in K_{i}} \sum_{m \in M_{i}^{k}} y_{i}^{k m} \leq \sum_{m \in M_{i}} c_{i}^{m} v_{i}^{m} \quad \forall i \in I
$$

is valid for CRPP and dominates set (1d).

Proof. If $\sum_{m \in M_{i}} v_{i}^{m}=0$, then $i$ is not sold and therefore $\sum_{k \in I^{k}} y_{i}^{k m}=0$. Otherwise, there exists $v_{i}^{m}=1$ for some $m \in M_{i}$, and $\sum_{k \in I^{k}} \sum_{m \in M_{i}^{k}} y_{i}^{k m} \leq \min \left\{c_{i},\left|\left\{k \in K_{i}: \sigma_{i}(k) \geq m\right\}\right|\right\}=$ : $c_{i}^{m}$. Therefore, (2) are valid.

Let us prove that they dominate $(1 \mathrm{~d})$. The fact that $c_{i}^{m} \leq c_{i} \forall m \in M_{i}$ implies:

$$
\sum_{k \in K_{i}} \sum_{m \in M_{i}^{k}} y_{i}^{k m} \leq \sum_{m \in M_{i}} c_{i}^{m} v_{i}^{m} \leq \sum_{m \in M_{i}} c_{i} v_{i}^{m} \leq c_{i} .
$$

Since sets (1d) and (2) have the same number of constraints, from now on we will consider formulation (3ICM) with (2) instead of (1d). Set (2) can be further strengthened:

Proposition 3.3. The set of constraints

$$
\sum_{\substack{k \in K_{i}: \\ \sigma_{i}(k) \geq m}} y_{i}^{k m} \leq c_{i}^{m} v_{i}^{m} \quad \forall i \in I, m \in M_{i}
$$

is valid for $\mathrm{CRPP}$ and dominates set (2). 
Proof. The proof of the validity of set (3) follows an analogous reasoning than that of set (2). The fact that (3) dominate (2) is clear, since we can obtain (2) from (3) summing up each size of the constraints for $m \in M_{i}$.

We can also strengthen sets $(1 \mathrm{~g})$ and (1i):

Proposition 3.4. The family of constraints

$$
c_{i} u_{i}^{m} \leq \sum_{\substack{k \in K_{i}: \\ \sigma_{i}(k) \geq m}} y_{i}^{k m} \quad \forall i \in I, m \in M_{i}
$$

is valid for CRPP and dominates family (1g).

Proof. If $u_{i}^{m}=0$ then the inequality holds trivially. Otherwise, $u_{i}^{m}=1$ means that product $i$ is sold out and that it has price $b_{i}^{m}$, so $\sum_{\substack{k \in K_{i}: \\ \sigma_{i}(k) \geq m}} y_{i}^{k m}=c_{i}$. The fact that (4) dominate $1 \mathrm{~g})$ is clear.

Proposition 3.5. The set of constraints

$$
v_{i}^{m} \leq u_{i}^{m}+y_{i}^{k m}+\sum_{\substack{j \in I: \\ j \prec k^{i}}} \sum_{m \in M_{j}^{k}} y_{j}^{k m} \quad \forall k \in K, i \in I^{k}, m \in M_{i}^{k}
$$

is valid for CRPP and dominates constraints (1i).

Proof. If $v_{i}^{m}=0$, then the inequality holds trivially. If $v_{i}^{m}=1$, then product $i$ is sold out (i.e. $u_{i}^{m}=1$ ) or $k$ purchases $i$ at price $b_{i}^{m}$ and $y_{i}^{k m}=1$, or $k$ purchases a product he prefers to $i$ (at any price) and thus $\sum_{\substack{j \in I: \\ j \prec k_{i}}} \sum_{m \in M_{j}^{k}} y_{j}^{k m}=1$. The fact that (5) dominate (1i) is straightforward considering (1f).

Proposition 3.6. The set of constraints

$$
\left(c_{i}-|S|\right) u_{i}^{m} \leq \sum_{\substack{k \in K_{i} \backslash S: \\ \sigma_{i}(k) \geq m}} y_{i}^{k m} \quad \forall i \in I, m \in M_{i}, S \subset K_{i}:|S|<c_{i}
$$

is valid for CRPP. If we set $S=\emptyset$, we obtain constraints (4).

Proof. If $u_{i}^{m}=0$, then the inequality holds trivially. If $u_{i}^{m}=1$, then product $i$ is sold out, so exactly $c_{i}$ copies are sold among customers from $S$ and from $K_{i} \backslash S$. In the worst case, every customer $k \in S$ has bought one copy of $i$, so at least $c_{i}-|S|$ copies of $i$ must be sold to customers from $K_{i} \backslash S$.

MET1 is the first method considered in the computational study presented in Section 6. and consists in formulation (3ICM). To assess the performance of the valid inequalities presented, we compare MET1 with MET2, where we solve formulation (3ICM) and include valid inequalities from (3), (4), (5) and (6) in a branch-and-cut fashion. Since sets (3)-(5) include a polynomial number of inequalities, we separate them by complete enumeration. 
As for set (6), it includes an exponential number of inequalities. To separate them, it is useful to notice that (6) come from the linearization of constraints

$$
c_{i} u_{i}^{m} \leq \sum_{k \in K_{i}^{m}} y_{i}^{k m} u_{i}^{m} \quad \forall i \in I, m \in M_{i}, k \in K_{i}^{m},
$$

where $K_{i}^{m}:=\left\{k \in K_{i}: \sigma_{i}(k) \geq m\right\}$.

In order to linearize (7), we must replace each non linear term of the RHS with an upper bound. Since all the variables are binary, it suffices to select one of the variables involved in each term. In this way, for any set $S \subset K_{i}$, we obtain the linear constraint $c_{i} u_{i}^{m} \leq$ $\sum_{k \in S} u_{i}^{m}+\sum_{k \in K_{i} \backslash S} y_{i}^{k m}$, which is precisely (6). From here, the separation procedure is simple. Given a fractional solution $\left(\bar{v}_{i}^{m}, \bar{y}_{i}^{k m}, \bar{u}_{i}^{m}\right)$ of (3ICM), for each $i$ and $m$ we consider set $S:=\left\{k \in K_{i}: \bar{u}_{i}^{m} \leq \bar{y}_{i}^{k m}\right\}$. The resultant constraint is added if and only if it is violated. Preliminary testing amounts for adding the violated cuts (from the four families) both at the root node and in the nodes of the branching tree of depth less than or equal to 3 .

\section{Two-index mixed integer formulation for the CRPP}

In this section, we introduce formulation (2ICM), that involves a much smaller number of variables than $(3 \mathrm{ICM})$.

As for variables, we use the sets $v_{i}^{m}$ and $u_{i}^{m}, \forall i \in I, m \in M_{i}$, previously introduced, and we define two more sets. In order to model the customers' purchasing decision, we define binary variable $x_{i}^{k}, \forall i \in I, k \in K_{i}$, that takes value 1 if customer $k$ purchases product $i$. Finally, in order to model a linear objective function, we define $z_{i}^{k}, \forall k \in K$, $i \in I^{k}$, as a continuous variable that takes as value the price of product $i$ if customer $k$ purchases it, and 0 if $k$ does not purchase it. Using these sets of variables, we introduce formulation (2ICM):

$$
\begin{aligned}
\text { (2ICM) } \max _{v, x, z, u} & \sum_{k \in K} \sum_{i \in I^{k}} z_{i}^{k} \\
\text { s.t. } \quad & \sum_{i \in I^{k}} x_{i}^{k} \leq 1 \quad \forall k \in K, \\
& \sum_{m \in M_{i}} v_{i}^{m} \leq 1 \quad \forall i \in I, \\
& \sum_{k \in K_{i}} x_{i}^{k} \leq \sum_{m \in M_{i}} c_{i}^{m} v_{i}^{m} \quad \forall i \in I, \\
& x_{i}^{k} \leq \sum_{m \in M_{i}^{k}} v_{i}^{m} \quad \forall k \in K, i \in I^{k}, \\
& u_{i}^{m} \leq v_{i}^{m} \quad \forall i \in I, m \in M_{i}, \\
& c_{i} \sum_{m \in M_{i}} u_{i}^{m} \leq \sum_{k \in K_{i}} x_{i}^{k} \quad \forall i \in I,
\end{aligned}
$$




$$
\begin{aligned}
& \sum_{m \in M_{i}} u_{i}^{m}+\left(c_{i}-1\right) \sum_{m \in M_{i}} v_{i}^{m} \geq \sum_{k \in K_{i}} x_{i}^{k} \quad \forall i \in I, \\
& \sum_{m \in M_{i}^{k}} v_{i}^{m} \leq \sum_{m \in M_{i}^{k}} u_{i}^{m}+\sum_{\substack{j \in I_{i} \\
j ﹎{k}}} x_{j}^{k} \quad \forall k \in K, i \in I^{k}, \\
& z_{i}^{k} \leq b_{i}^{\sigma_{i}(k)} x_{i}^{k} \quad \forall k \in K, i \in I^{k}, \\
& z_{i}^{k} \leq \sum_{m \in M_{i}^{k}} b_{i}^{m} v_{i}^{m} \quad \forall k \in K, i \in I^{k}, \\
& v_{i}^{m}, u_{i}^{m} \in\{0,1\} \quad \forall i \in I, m \in M_{i}, \\
& x_{i}^{k} \in\{0,1\} \quad \forall k \in K, i \in I^{k}, \\
& z_{i}^{k} \geq 0 \quad \forall k \in K, i \in I^{k} .
\end{aligned}
$$

Constraints (8b) guarantee that customers purchase at most one product from the company. Constraints (8c) ensure that each product has at most one price. Constraints (8d) are the capacity constraints, and they assure that no more than $c_{i}$ copies of product $i$ are sold. Constraints (8e) prevent customer $k$ from purchasing product $i$ if he cannot afford it. Constraints $(8 \mathrm{f})$ - $(8 \mathrm{~h})$ force the $u$-variables to take their right value: $(8 \mathrm{f})$ establish that a product $i$ can only sell out at price $b_{i}^{m}$ (i.e. $u_{i}^{m}=1$ ) if it has such price; $(8 \mathrm{~g})$ force $u_{i}^{m}$ to be 0 when $\sum_{k \in K_{i}} x_{i}^{k}<c_{i}$; and (8h) imply $u_{i}^{m}=1$ when $\sum_{k \in K_{i}} x_{i}^{k}=c_{i}$. Constraints 8i are the preference constraints and guarantee that the preferences are satisfied when the products are not sold out. Note that when $\sum_{m \in M_{i}^{k}} v_{i}^{m}=1$, customer $k$ can afford product $i$. Therefore, either $\sum_{m \in M_{i}^{k}} u_{i}^{m}=1$, that is, the product is sold out, or customer $k$ must purchase $i$ or a product he prefers over $i$, so $\sum_{\substack{j \in I \\ j \leq k_{i}}} x_{j}^{k}=1$. The last sets of constraints (8j)-8k bound the $z$-variables to their value. If customer $k$ does not purchase product $i$, then by (8j) we deduce $z_{i}^{k}=0$. On the other hand, if $x_{i}^{k}=1$, then the RHS of (8j) is an upper bound on $z_{i}^{k}$, and $k$ is able to afford $i$, so $v_{i}^{\bar{m}}=1$ for some $\bar{m} \leq \sigma_{i}(k)$. But then by $(8 \mathrm{k})$ we obtain that $z_{i}^{k} \leq b_{i}^{\bar{m}}$, which is exactly the price of $i$. Finally, the objective function (8a) is the sum of the revenue of the company obtained for each customer $k$ and product $i$.

Much like (3ICM), formulation (2ICM) can be strengthened adding valid inequalities. In the following section, we include three sets of valid inequalities developed for (2ICM).

\section{Valid inequalities for formulation (2ICM) and res- olution schemes}

In this section, we introduce three families of valid inequalities for formulation (2ICM). In the last subsection, we also explain the separation procedures developed to include them and the branch-and-cut algorithms. We have also included an in-out stabilization procedure to avoid the tailing off effect. In Section 6, we test and compare the performance of these resolution schemes. 


\subsection{Projecting out the customer decision variables}

Formulation (3ICM) has a larger number of variables and constraints than (2ICM), but the linear relaxation of (3ICM) with the valid inequalities (3)-(6) gives a stronger upper bound. In this subsection, we discuss how to project out formulation (3ICM) on formulation (2ICM), obtaining a subproblem that can be solved for a given fractional feasible solution of (2ICM) to derive valid inequalities.

First of all, let us extend formulation (2ICM) adding the $y$-variables used in (3ICM), as well as the necessary constraints to relate them to the rest of the variables. By definition, $x_{i}^{k}=\sum_{m \in M_{i}} y_{i}^{k m}$ and $z_{i}^{k}=\sum_{m \in M_{i}} b_{i}^{m} y_{i}^{k m}$ for all $k \in K, i \in I^{k}$. Adding the valid inequalities developed in Section 3, we obtain:

$$
\begin{aligned}
(2 \mathrm{ICM}+) \quad \max _{v, y, x, z, u} & \sum_{k \in K} \sum_{i \in I^{k}} z_{i}^{k} \\
\text { s.t. } & (8 \mathrm{~b})-(8 \mathrm{n}),(1 \mathrm{e}),(1 \mathrm{k}),(3),(4), \\
& v_{i}^{m} \leq u_{i}^{m}+y_{i}^{k m}+\sum_{\substack{j \in I_{i} \\
j \prec k^{i}}} x_{j}^{k} \quad \forall k \in K, i \in I^{k}, m \in M_{i}^{k}, \\
& z_{i}^{k} \leq \sum_{m \in M_{i}^{k}} b_{i}^{m} y_{i}^{k m} \quad \forall k \in K, i \in I^{k}, \\
& x_{i}^{k} \geq \sum_{m \in M_{i}^{k}} y_{i}^{k m} \quad \forall k \in K, i \in I^{k} .
\end{aligned}
$$

Constraints $(8 \mathrm{~b})-(8 \mathrm{n})$ along with the objective function constitute formulation (2ICM). Constraints (1e), (3), (4) and (9a) dominate, respectively, constraints (8e), (8d), (8g) and 8i). In fact, constraints (9a) are (5) with $x_{j}^{k}$ replacing $\sum_{m \in M \in M_{j}^{k}} y_{j}^{k m}$ in the third sum. Finally, constraints (9b) and (9c) relate the $y$-variables with the $x$ - and $z$-variables. Although $(9 \mathrm{~b})$ appear as inequalities, they are satisfied as equalities by any optimal solution of $(2 \mathrm{ICM}+)$ due to the objective function. As for (9c), let us assume that there exists a feasible solution with $x_{i}^{k}>\sum_{m \in M_{i}^{k}} y_{i}^{k m}$. Then since the variables are binary, it follows $x_{i}^{k}=1$ and $\sum_{m \in M_{i}^{k}} y_{i}^{k m}=0$. But then $\sum_{m \in M_{i}^{k}} y_{i}^{k m}=0 \Rightarrow \sum_{m \in M_{i}^{k}} b_{i}^{m} y_{i}^{k m}=0$, so by $9 \mathrm{~b}$ we obtain $z_{i}^{k}=0$. Therefore $k$ is purchasing product $i$ but the revenue of the company associated to this customer is 0 , so the solution is not optimal.

Proposition 5.1. Consider a fixed product $i \in I$. Then the following family of constraints

$$
\begin{aligned}
\sum_{k \in K_{i}} z_{i}^{k} \alpha^{k}+\sum_{m \in M_{i}} c_{i} u_{i}^{m} \lambda^{m}+\sum_{k \in K_{i}} \sum_{m \in M_{i}^{k}}\left(v_{i}^{m}-u_{i}^{m}+\sum_{\substack{j \in I_{i} \\
j \prec k^{i}}} x_{i}^{k}\right) \epsilon^{k m} \leq \\
\sum_{k \in K_{i}} x_{i}^{k} \beta^{k}+\sum_{k \in K_{i}} \sum_{m \in M_{i}^{k}} v_{i}^{m} \gamma^{k m}+\sum_{m \in M_{i}} c_{i}^{m} v_{i}^{m} \delta^{m}
\end{aligned}
$$

is valid for (2ICM) if for $\alpha^{k}, \beta^{k} \geq 0 \forall k \in K_{i}, \gamma^{k m}, \epsilon \geq 0 \forall k \in K_{i}, m \in M_{i}^{k}$ and $\delta^{m}, \lambda^{m} \geq 0, \forall m \in M_{i}^{k}$, it holds

$$
b_{i}^{m} \alpha^{k}+\lambda^{m}+\epsilon^{k m} \leq \beta^{k}+\gamma^{k m}+\delta^{m} \quad \forall k \in K_{i}, m \in M_{i}^{k} .
$$


Proof. For a fixed $i \in I$, we associate dual variables $\alpha^{k}, \beta^{k}, \gamma^{k m}, \delta^{k}, \lambda^{m}$ and $\epsilon^{k m}$ to constraints (9b), (9c), (1e), (3), (4) and (9a), respectively. Then the result follows applying Farkas' Lemma.

As for constraints (6), they cannot be projected out because the set includes an exponential number. However, an alternative consists in developing an equivalent set of valid inequalities for (2ICM). Thus, we can linearize constraints

$$
c_{i} u_{i}^{m} \leq \sum_{k \in K_{i}^{m}} x_{i}^{k} v_{i}^{m} u_{i}^{m} \quad \forall i \in I, m \in M_{i}, k \in K_{i}^{m},
$$

with $K_{i}^{m}:=\left\{k \in K_{i}: \sigma_{i}(k) \geq m\right\}$, in a similar manner to family (7), obtaining

$$
c_{i} u_{i}^{m} \leq \sum_{k \in S_{1}} u_{i}^{m}+\sum_{k \in S_{2}} v_{i}^{m}+\sum_{\substack{k \in K_{i} \backslash \\\left(S_{1} \cup S_{2}\right)}} x_{i}^{k}, \quad \forall i \in I, m \in M_{i}, S_{1}, S_{2} \subset K_{i}: S_{1} \cap S_{2}=\emptyset \text {. }
$$

Family (13) can be reduced noticing that the subfamily where $S_{2}=\emptyset$ dominates (13) due to $(8 \mathrm{f})$. Nonetheless, preliminary testing showed that the inclusion of valid inequalities from this family does not improve the linear relaxation bound from (2ICM), so (13) is not included in the computational experiments of Section 6 .

\subsection{Separation of valid inequalities from family 10}

In what follows, we are going to analyze the problem that results from fixing $\lambda=\delta=\epsilon=0$ in (10). The reason is that the inequalities associated to variables $\alpha, \beta$ and $\gamma$ can be separated by customer (as well as by product). Next, we study the particular case $\alpha=1$. Thus, in order to obtain the strongest bound we look for values of $\alpha$ and $\beta$ which provide the smaller value of the RHS of (10). Considering a fixed product $i$ and customer $k$, our problem (SP1) can be stated as:

$$
\begin{array}{ll}
\min _{\beta, \gamma} & x_{i}^{k} \beta+\sum_{m \in M_{i}^{k}} v_{i}^{m} \gamma^{m} \\
\text { s.t. } & \beta+\gamma^{m} \geq b_{i}^{m} \quad \forall m \in M_{i}^{k}, \\
& \beta, \gamma \geq 0 .
\end{array}
$$

For a given $\beta$, constraints $(14 \mathrm{~b})$ can be expressed as $\gamma^{m} \geq b_{i}^{m}-\beta$ for each $m$. Hence, $\gamma^{m}=\max \left\{0, b_{i}^{m}-\beta\right\}$. Let us see that the best value for $\beta$ belongs to the set of candidate reservation prices $\left\{b_{i}^{1}, \ldots, b_{i}^{\sigma_{i}(k)}\right\}$. Suppose that $b_{i}^{r}<\beta<b_{i}^{r+1}$. Then $\gamma^{m}=0$ for $m \leq r$, and $\gamma^{m}=b_{i}^{r}-\beta$ for $m>r$. Substituting $\gamma$ in the objective function of (SP1), we obtain:

$$
x_{i}^{k} \beta+\sum_{\substack{m \in M_{i}^{k}: \\ m>r}}\left(b_{i}^{r}-\beta\right) v_{i}^{m}=\left(x_{i}^{k}-\sum_{\substack{m \in M_{i}^{k}: \\ m>r}} v_{i}^{m}\right) \beta+\sum_{\substack{m \in M_{i}^{k}: \\ m>r}} b_{i}^{r} v_{i}^{m} .
$$

The above linear function in $\beta$ attains its minimum value in one of its extreme values, that is, $\beta=b_{i}^{r}$ or $\beta=b_{i}^{r+1}$, depending on the sign of the slope $x_{i}^{k}-\sum_{\substack{m \in M_{i}^{k} \\ m>r}} v_{i}^{m}$. This leads to the following 
Proposition 5.2. The family of inequalities

$$
z_{i}^{k} \leq b_{i}^{r_{i}^{k}} x_{i}^{k}+\sum_{m=r_{i}^{k}+1}^{\sigma_{i}(k)}\left(b_{i}^{m}-b_{i}^{r_{i}^{k}}\right) v_{i}^{m}, \quad \forall k \in K, i \in I^{k}, r_{i}^{k} \in\left\{0, \ldots, \sigma_{i}(k)\right\}
$$

is valid for (2ICM).

Family (16) is included in a family of valid inequalities of exponential size originally developed in [5] for the RPP (the uncapacitated version):

$$
z_{i}^{k} \leq b_{i}^{r_{i}^{k}} x_{i}^{k}+\sum_{m=r_{i}^{k}+1}^{\sigma_{i}(k)}\left(b_{i}^{m}-b_{i}^{r_{i}^{k}}\right) v_{i}^{m}+\sum_{m \in Q_{i}^{k}}\left(b_{i}^{m}-b_{i}^{r_{i}^{k}}\right)\left(x_{i}^{k}+v_{i}^{m}-1\right),
$$

$\forall k \in K, i \in I^{k}$, any integer $r_{i}^{k} \in\left\{0, \ldots, \sigma_{i}(k)\right\}$ and any subset $Q_{i}^{k} \subseteq\left\{1, \ldots, r_{i}^{k}-1\right\}$. In the following, we prove that family (16) dominates (17).

Proposition 5.3. Given $k \in K, i \in I^{k}$, the strongest valid inequality from family (17) is obtained when $Q_{i}^{k}=\emptyset$.

Proof. Let us consider $k \in K, i \in I^{k}$ fixed and, for the sake of notation, $r:=r_{i}^{k}$ and $Q:=Q_{i}^{k}$. First of all, let $q:=\min _{Q}\{m\}$ (and therefore, $q<r$ and $b_{i}^{q}<b_{i}^{r}$ ). Then the following inequality

$$
z_{i}^{k} \leq b_{i}^{r} x_{i}^{k}+\sum_{m=q}^{\sigma_{i}(k)}\left(b_{i}^{m}-b_{i}^{r}\right) v_{i}^{m}+\left(b_{i}^{q}-b_{i}^{r}\right)\left(x_{i}^{k}-1\right)
$$

is valid and stronger than the corresponding inequality of (17), for all $r$ and $Q$.

Let us prove its validity first. If $x_{i}^{k}=1$, then $v_{i}^{m}=1$ for some $\bar{m} \leq \sigma_{i}(k)$, and the inequality becomes $z_{i}^{k} \leq b_{i}^{r}+\left(b_{i}^{\bar{m}}-b_{i}^{r}\right)=b_{i}^{\bar{m}}$, valid because the price of product $i$ is exactly $b_{i}^{\bar{m}}$. On the other hand, if $x_{i}^{k}=0$, then the inequality becomes $z_{i}^{k} \leq \sum_{m=q}^{\sigma_{i}(k)}\left(b_{i}^{m}-b_{i}^{r}\right) v_{i}^{m}+$ $b^{r}-b^{q}$. If $v_{i}^{m_{0}}=1$ for $m_{0} \geq r$, then the first sum of the RHS is non negative. If $v_{i}^{m_{0}}=1$ for $m_{0}<r$, then the inequality is $z_{i}^{k} \leq b_{i}^{m_{0}}-b_{i}^{r}+b_{i}^{r}-b_{i}^{q}=b_{i}^{m_{0}}-b_{i}^{q}$, non negative because of the definition of $q$. In both cases, since $z_{i}^{k}=0$ and the RHS of the inequality is greater than or equal to zero, the inequality holds.

Now, to prove that it is stronger, let us subtract the RHS of 18 from the RHS of (17) to see that the difference is non negative:

$$
\begin{aligned}
& \left(b_{i}^{r} x_{i}^{k}+\sum_{m=r+1}^{\sigma_{i}(k)}\left(b_{i}^{m}-b_{i}^{r}\right) v_{i}^{m}+\sum_{m \in Q}\left(b_{i}^{m}-b_{i}^{r}\right)\left(x_{i}^{k}+v_{i}^{m}-1\right)\right)- \\
& \left(b_{i}^{r} x_{i}^{k}+\sum_{m=q}^{\sigma_{i}(k)}\left(b_{i}^{m}-b_{i}^{r}\right) v_{i}^{m}+\left(b_{i}^{q}-b_{i}^{r}\right)\left(x_{i}^{k}-1\right)\right)= \\
& =\sum_{m \in\{q, \ldots, r-1\} \backslash Q}\left(b_{i}^{m}-b_{i}^{r}\right) v_{i}^{m}-\max _{m \in Q \backslash\{q\}}\left\{b_{i}^{m}-b_{i}^{r}\right\}\left(x_{i}^{k}-1\right) \geq 0 .
\end{aligned}
$$


In the following, let us consider $W(r)$ as the RHS of (18) with $q$ fixed to study its variation when $r$ increases:

$$
\begin{aligned}
W(r+1)-W(r) & =\left(b_{i}^{r+1}-b_{i}^{r}\right) x_{i}^{k}+\sum_{m=q}^{\sigma_{i}(k)}\left(b_{i}^{r+1}-b_{i}^{r}\right) v_{i}^{m}+\left(b^{-} r_{i}-b_{i}^{r+1}\right)\left(x_{i}^{k}-1\right)= \\
& =\left(b_{i}^{r+1}-b_{i}^{r}\right)\left(1-\sum_{m=q}^{\sigma_{i}(k)} v_{i}^{m}\right) \geq 0 .
\end{aligned}
$$

Supposing $q$ is fixed yields a non negative difference whenever we increment $r$, so the optimal is to select the smallest possible $r$. Given that $q \leq r$, we will hence choose $r=q$. This implies that $Q=\emptyset$, and we have proved the statement.

\subsection{An additional set of valid inequalities}

In this subsection, we present another set of valid inequalities developed for formulation (2ICM). Unlike set (16), this set includes one inequality per product, and in this case the capacity is used to strengthen the inequalities.

Proposition 5.4. Consider the set of inequalities

$$
\begin{gathered}
\sum_{k \in K_{i}} z_{i}^{k}+\sum_{m \in M_{i}} \sum_{m^{\prime}=m+1}^{\left|M_{i}\right|}\left(b_{i}^{m^{\prime}}-b_{i}^{m^{\prime}-1}\right) U_{i}^{m m^{\prime}} u_{i}^{m} \leq \\
\sum_{k \in K_{i}} b_{i}^{r^{k}} x_{i}^{k}+\sum_{m \in M_{i}} \sum_{m^{\prime}=1}^{m}\left(b_{i}^{m^{\prime}}-b_{i}^{m^{\prime}-1}\right) V_{i}^{m m^{\prime}} v_{i}^{m},
\end{gathered}
$$

$\forall i \in I, r^{k} \in\left\{0, \ldots, \sigma_{i}(k)\right\} \forall k \in K_{i}$, where $U_{i}^{m m^{\prime}}:=\max \left\{0, c_{i}-\mid\left\{k \in K_{i}: \sigma_{i}(k) \geq m\right.\right.$, $\left.\left.r^{k}<m^{\prime}\right\} \mid\right\}$ and $V_{i}^{m m^{\prime}}:=\min \left\{c_{i}^{m}, \mid\left\{k \in K_{i}: \sigma_{i}(k) \geq m, r^{k}<m^{\prime} \mid\right\}\right\}, \forall i \in I, m \in M_{i}$, $m^{\prime} \in M_{i}$. Set 20) is valid for (2ICM).

Proof. Let $i \in I$ be a fixed product and let $r^{k} \in\left\{0, \ldots, \sigma_{i}(k)\right\}$ be fixed reservation prices indices $\forall k \in K_{i}$. Let $(\overline{\boldsymbol{v}}, \overline{\boldsymbol{x}}, \overline{\boldsymbol{z}}, \overline{\boldsymbol{u}})$ be a feasible (integer) solution of formulation (2ICM). If $\sum_{m \in M_{i}} \bar{v}_{i}^{m}=0$, then the corresponding inequality holds trivially because all the variables have value zero. Therefore, we assume $\bar{v}_{i}^{\bar{m}}=1$ for some $\bar{m} \in M_{i}$. This yields $\bar{u}_{i}^{m}=0$ for $m \neq \bar{m}$, and $\bar{u}_{i}^{\bar{m}} \in\{0,1\}$.

First of all, let $\bar{K}_{i}:=\left\{k \in K_{i}: \bar{x}_{i}^{k}=1\right\}$ be the set of customers purchasing $i$. Then the following inequality holds:

$$
\sum_{k \in K_{i}} \bar{z}_{i}^{k}+\sum_{m \in M_{i}} \sum_{\substack{k \in \bar{K}_{i}: \\
r^{k}>m}}\left(b_{i}^{r^{k}}-b_{i}^{m}\right) \bar{u}_{i}^{m} \leq \sum_{k \in K_{i}} b_{i}^{r^{k}} \bar{x}_{i}^{k}+\sum_{\substack { m \in M_{i} \\
\begin{subarray}{c}{k \in \bar{K}_{i}: \\
r^{k}<m{ m \in M _ { i } \\
\begin{subarray} { c } { k \in \overline { K } _ { i } : \\
r ^ { k } < m } }\end{subarray}}\left(b_{i}^{m}-b_{i}^{r^{k}}\right) \bar{v}_{i}^{m} .
$$

Indeed, if $\bar{u}_{i}^{\bar{m}}=1$ the LHS of (21) is equal to $\sum_{k \in \bar{K}_{i}} b_{i}^{\bar{m}}+\sum_{k \in \bar{K}_{i}: r^{k}>\bar{m}}\left(b_{i}^{r^{k}}-b_{i}^{\bar{m}}\right)$, which is equal to its RHS, $\sum_{k \in \bar{K}_{i}} b_{i}^{r^{k}}+\sum_{k \in \bar{K}_{i}: r^{k}<\bar{m}}\left(b_{i}^{\bar{m}}-b_{i}^{r^{k}}\right)$. Otherwise, the LHS is 
$\sum_{k \in \bar{K}_{i}} b_{i}^{\bar{m}}$ and thus it also holds. Now, in order to see that the statement holds, we shall prove that the LHS (resp. RHS) of (20) is smaller than or equal to (resp. greater than or equal to) the LHS (resp. the RHS) of (21). Given that $\bar{u}_{i}^{m}=\bar{v}_{i}^{m}=0 \forall m \neq \bar{m}$ and $\bar{v}_{i}^{\bar{m}}=1$, this translates to proving:

$$
\sum_{\substack{k \in \bar{K}_{i}: \\ r^{k}<\bar{m}}}\left(b_{i}^{\bar{m}}-b_{i}^{r^{k}}\right) \leq \sum_{m^{\prime}=1}^{\bar{m}}\left(b_{i}^{m^{\prime}}-b_{i}^{m^{\prime}-1}\right) V_{i}^{\bar{m} m^{\prime}}
$$

$$
\sum_{\substack{k \in \bar{K}_{i}: \\ r^{k}>\bar{m}}}\left(b_{i}^{r^{k}}-b_{i}^{\bar{m}}\right) \geq \sum_{m^{\prime}=\bar{m}+1}^{\left|M_{i}\right|}\left(b_{i}^{m^{\prime}}-b_{i}^{m^{\prime}-1}\right) U_{i}^{\bar{m} m^{\prime}} \text { if } \bar{u}_{i}^{\bar{m}}=1 .
$$

To prove (1), we have

$$
\begin{aligned}
& \sum_{\substack{k \in \bar{K}_{i}: \\
r^{k}<\bar{m}}}\left(b_{i}^{\bar{m}}-b_{i}^{r^{k}}\right)=\sum_{\substack{k \in \bar{K}_{i}: \\
r^{k}<\bar{m}}} \sum_{m^{\prime}=r^{k}+1}^{\bar{m}}\left(b_{i}^{m^{\prime}}-b_{i}^{m^{\prime}-1}\right)=\sum_{m^{\prime}=1}^{\bar{m}}\left(b_{i}^{m^{\prime}}-b_{i}^{m^{\prime}-1}\right) \sum_{\substack{k \in \bar{K}_{i}: \\
r^{k}<m^{\prime}}} 1 \\
& =\sum_{m^{\prime}=1}^{\bar{m}}\left(b_{i}^{m^{\prime}}-b_{i}^{m^{\prime}-1}\right)\left|\left\{k \in \bar{K}_{i}: r^{k}<m^{\prime}\right\}\right|,
\end{aligned}
$$

where the second equality is obtained switching the sums in $m^{\prime}$ and $m$. Thus, the fact that $\left|\bar{K}_{i}\right| \leq c_{i}^{\bar{m}}$ and that for all $k \in \bar{K}_{i}$ it holds $\sigma_{i}(k) \geq \bar{m}$ implies $\left|\left\{k \in \bar{K}_{i}: r^{k}<m^{\prime}\right\}\right| \leq$ $\min \left\{c_{i}^{\bar{m}},\left|k \in K_{i}: \sigma_{i}(k) \leq \bar{m}, r^{k}<m^{\prime}\right|\right\}=: V_{i}^{\bar{m} m^{\prime}}$, and (1) holds.

To prove (2), we follow an analogous procedure:

$$
\begin{aligned}
& \sum_{\substack{k \in \bar{K}_{i}: \\
r^{k}>\bar{m}}}\left(b_{i}^{r^{k}}-b_{i}^{\bar{m}}\right)=\sum_{\substack{k \in \bar{K}_{i}: \\
r^{k}>\bar{m}}} \sum_{m^{\prime}=\bar{m}+1}^{r^{k}}\left(b_{i}^{m^{\prime}}-b_{i}^{m^{\prime}-1}\right)=\sum_{m^{\prime}=\bar{m}+1}^{\left|M_{i}\right|}\left(b_{i}^{m^{\prime}}-b_{i}^{m^{\prime}-1}\right) \sum_{\substack{k \in \bar{K}_{i}: \\
r^{k} \geq m^{\prime}}} 1 \\
& =\sum_{m^{\prime}=\bar{m}+1}^{\left|M_{i}\right|}\left(b_{i}^{m^{\prime}}-b_{i}^{m^{\prime}-1}\right)\left|\left\{k \in \bar{K}_{i}: r^{k} \geq m^{\prime}\right\}\right| .
\end{aligned}
$$

The result follows because $\left|\left\{k \in \bar{K}_{i}: r^{k} \geq m^{\prime}\right\}\right|=\left|\bar{K}_{i}\right|-\left|\left\{k \in \bar{K}_{i}: r^{k}<m^{\prime}\right\}\right|=$ $c_{i}-\left|\left\{k \in \bar{K}_{i}: \sigma_{i}(k) \geq \bar{m}, r^{k}<m^{\prime}\right\}\right| \geq c_{i}-\left|\left\{k \in K_{i}: \sigma_{i}(k) \geq \bar{m}, r^{k}<m^{\prime}\right\}\right|$, and therefore $\left|\left\{k \in \bar{K}_{i}: r^{k} \geq m^{\prime}\right\}\right| \geq U_{i}^{\bar{m} m^{\prime}}$.

Method MET3 consists in solving an instance of the CRPP using formulation (2ICM). We have developed four additional methods based on (2ICM), that are fully detailed in the following subsection.

\subsection{Separation algorithms and resolution schemes using formu- lation (2ICM) and an in-out stabilization method}

We begin this subsection with the introduction of the three separation procedures designed to include valid inequalities (10), (16) and (20) dynamically into formulation (2ICM). 
Then, we show the four resolution methods developed and the in-out stabilization method proposed. The first three methods (MET4-MET6) correspond to a branch-and-cut involving valid inequalities from each of the three families presented, namely (10), (16) and (20). The last one (MET7) is a branch-and-cut including a combination of valid inequalities from families (16) and (20). These approaches are compared in Section 6 using extensive computational experiments.

The first method MET4 incorporates violated inequalities from (10) to formulation (2ICM). In order to obtain the dual variables for the inequalities in (10), we solve the following separation problem $\left(S P_{i}\right)$ (by means of a commercial solver) for each product $i \in I$ :

$$
\begin{aligned}
\max _{\alpha, \boldsymbol{\beta}, \boldsymbol{\gamma}, \boldsymbol{\delta}, \boldsymbol{\lambda}} & \sum_{k \in K_{i}} z_{i}^{k} \alpha^{k}+\sum_{m \in M_{i}} c_{i} u_{i}^{m} \lambda^{m}-\sum_{k \in K_{i}} x_{i}^{k} \beta^{k}-\sum_{k \in K_{i}} \sum_{m \in M_{i}^{k}} v_{i}^{m} \gamma^{k m}-\sum_{m \in M_{i}} c_{i} v_{i}^{m} \delta^{m} \\
\text { s.t. } & b_{i}^{m} \alpha^{k}+\lambda^{m} \leq \beta^{k}+\gamma^{k m}+\delta^{m} \quad \forall k \in K_{i}, m \in M_{i}^{k}, \\
& \alpha^{k}, \beta^{k} \geq 0 \quad \forall k \in K_{i}, \\
& \gamma^{k m} \geq 0 \quad \forall k \in K_{i}, m \in M_{i}^{k}, \\
& \delta^{m}, \lambda^{m} \geq 0 \quad \forall m \in M_{i} .
\end{aligned}
$$

Cuts from (10) are included after the linear relaxation of (2ICM), until no more valid inequalities are violated or the linear relaxation bound does not decrease, and in the nodes of the branching tree. Preliminary testing led us to include valid inequalities during the branch-and-bound in the nodes of depth less than or equal to 3.

Method MET5 requires a separation procedure to include inequalities from (16) dynamically in a branch-and-cut framework. Thus, if we are given a fractional feasible solution of formulation (2ICM), we calculate the $r$ that minimizes the RHS of (16) for each $k$ and $i$ fixed simplifying the reasoning developed in [5] for (17). In essence, we study the variation of the RHS of (16) as $r$ increases. Calling $W(r)$ the RHS of $(16)$ for a given $r$, we have

$$
W(r+1)-W(r)=\left(b^{r+1}-b^{r}\right)\left(\bar{x}_{i}^{k}-\sum_{m=r+1}^{\sigma_{i}(k)} \bar{v}_{i}^{m}\right)
$$

Clearly $b^{r+1}-b^{r}>0$, and the sum $\sum_{m=r+1}^{\sigma_{i}(k)} \bar{v}_{i}^{m}$ has less terms as $r$ increases. Therefore, the slope $\bar{x}_{i}^{k}-\sum_{m=r+1}^{\sigma_{i}(k)} \bar{v}_{i}^{m}$ is negative and for some $r$, the slope changes to a positive value. So the minimum in 23 is obtained for the $r$ such that $x_{i}^{k}-\sum_{m=r+1}^{\sigma_{i}(k)} v_{i}^{m} \leq 0$ and $x_{i}^{k}-\sum_{m=r+2}^{\sigma_{i}(k)} v_{i}^{m}>0$. Algorithm 1 depicts the separation used in MET5 to incorporate inequalities from set 16 in a branch-and-cut fashion.

The third method MET6 consists in solving (2ICM) including valid inequalities from set 20) in a branch-and-cut. MET6 also requires a separation algorithm for set 20), but this algorithm is very similar to 1 . The calculation of $r^{k} \forall k \in K_{i}$ is analogous to that depicted in Algorithm 1. In this case, at each iteration in a node of the branching tree, we include one inequality per product (if violated). Like in MET3, inequalities from (20) are also included when fractional solutions are found in the nodes of the branching tree of depth smaller than or equal to three. 


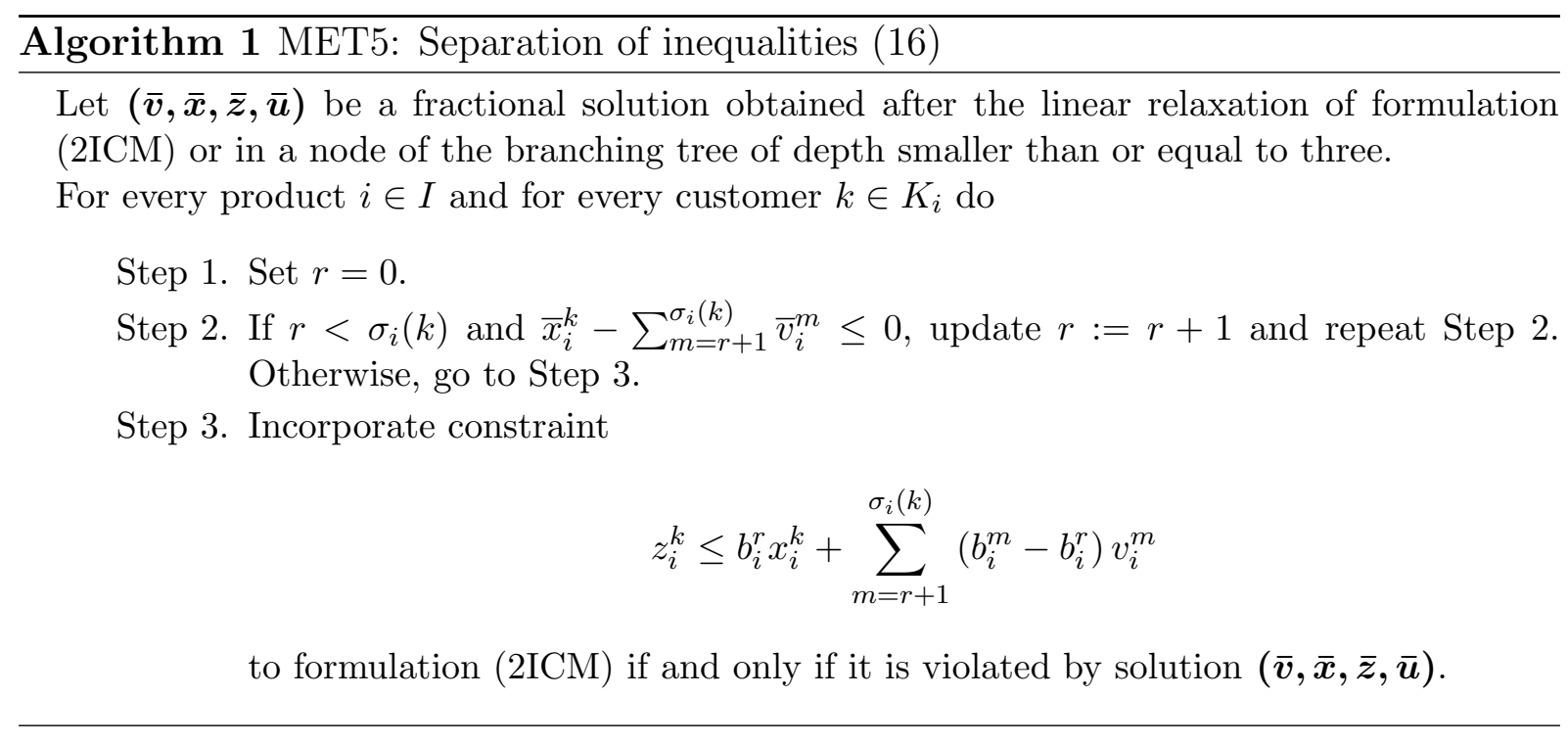

When valid inequalities from set (16) are separated, we include one inequality per customer and product at each iteration. However, in the case of inequalities from family (20), only one inequality per product is included at each step, and each of them combines variables associated to all the customers. Therefore, valid inequalities from 20 have a much larger number of non zero elements than those from (16). Moreover, and according to preliminary testing, the linear relaxation bound of model (2ICM) improves slowly when adding inequalities from (20), so a great number of iterations is required in this case. These are the reasons for incorporating an in-out algorithm to stabilize the inclusion of inequalities in the root node in the last two methods MET6 and MET7. This algorithm has been developed by Ben-Ameur and Neto [2], used for other capacitated problems (e.g. in Fischetti et al. [10]) and it is widely used to include Benders decomposition cuts. It is of particular importance when only a few valid inequalities are separated at each iteration, like in this case. It allows to generate less cuts of better quality, hopefully reducing the tailing-off effect. In the following we describe the in-out procedure.

Let the polyhedron $D^{\prime}$ defined by the linear relaxation of $(2 \mathrm{ICM})$ and $D \subset D^{\prime}$ the polyhedron defined by this same linear relaxation plus family (20). Consider a point $\rho_{\text {out }} \in D^{\prime} \backslash D$ which is the optimal solution of the linear relaxation of (2ICM). A separation algorithm is used to derive an inequality (from (20) ) separating a given separation point from $D$. The classical approach sets $\rho_{\text {out }}$ as the separation point, whereas the in-out algorithm provides a different separation point $\rho_{\text {sep }}$. To derive it, define an initial feasible interior point $\rho_{\text {in }} \in D$ and create a convex combination of points $\rho_{\text {in }}$ and $\rho_{\text {out }}$, obtaining $\rho_{\text {sep }}=\lambda \rho_{\text {out }}+(1-\lambda) \rho_{\text {in }}$, with $\lambda \in(0,1]$. Thus, when we call the separation oracle for $\rho_{\text {sep }}$ we have two possible outcomes, depending on whether $\rho_{\text {sep }} \in D$ or $\rho_{\text {sep }} \notin D$. If we obtain violated cuts, then $\rho_{\text {sep }} \notin D$, so after including such cuts and reoptimizing the linear relaxation of our current problem, we obtain a new point $\rho_{\text {out }}$. On the other hand, if no valid inequalities are found, then $\rho_{\text {sep }} \in D$ and we can use $\rho_{\text {sep }}$ as the interior point $\rho_{\text {in }}$ in the following iteration. Hence, either $\rho_{\text {in }}$ or $\rho_{\text {out }}$ are updated at each iteration, and the termination criteria defined is $\left|\rho_{\text {in }}-\rho_{\text {out }}\right|<\epsilon$. Although $\lambda$ is a scalar that can change in every iteration, preliminary testing led us to set $\lambda=0.99$ for all iterations. As for the initial interior point $\rho_{\text {in }}$, it is frequently obtained using the barrier algorithm with crossover. In our case, we built a non-degenerate convex combination of enough linearly 


\begin{tabular}{l|l} 
Name & Description \\
\hline MET1 & $(3 \mathrm{ICM})$ \\
MET2 & $(3 \mathrm{ICM})+$ branch-and-cut with vv.ii. from sets (3), (4), (5) and (6) \\
MET3 & $(2 \mathrm{ICM})$ \\
MET4 & $(2 \mathrm{ICM})+$ branch-and-cut with vv.ii. from set (10) \\
MET5 & $(2 \mathrm{ICM})+$ branch-and-cut with vv.ii. from set (16) \\
MET6 & $(2 \mathrm{ICM})+$ branch-and-cut with vv.ii. from set (20) \\
MET7 & $(2 \mathrm{ICM})+$ branch-and-cut with vv.ii. from sets $(16)$ and $(20)$
\end{tabular}

Table 1: Details of methods MET1-MET7 tested in the computational study

independent points of the polytope and then obtained the centroid.

Finally, in MET7 we separate inequalities from families (16) and (20). Through preliminary computational experiments, we observed that the upper bound of the linear relaxation obtained separating inequalities from (20) was tighter than that obtained with valid inequalities from (16). However, inequalities from (16) require less iterations to reduce the bound. To combine them in the cut loop previous to the branching, we begin including only constraints from (16) until no more are found. Then, we include only inequalities from (20) and further reduce the bound. The separation oracles are the ones previously described, and the in-out algorithm is included in this step of MET7. We also separate inequalities from both sets in all the fractional solutions obtained in the nodes of the branching tree of depth less than or equal to three (including all the violated ones from both sets at every node).

We have depicted the seven resolution methods developed and their characteristics in Table 1, to help to identify them easily.

\section{Computational study}

In this section, we compare the performance of formulations (2ICM) and (3ICM) and the valid inequalities proposed testing the methods MET1-MET7. The computational experiments were carried out on a personal computer with Intel Xeon E3-1270, 3.40 GHz with 16 GB of RAM. The optimization problems were solved exactly by using the solver Xpress Optimizer Version: 29.01.10 and the methods were coded using Mosel Version: 4.0 .3 .

To test the algorithms, we created instances with $|K|=50,|K|=75,|K|=100$, $|K|=125$ and $|K|=150$ customers. For each size of set $K$, we designed instances with $|I|=5,|I|=10$ and $|I|=15$ products. In the computational studies carried out for the RPP ([5]) and the RPPT ([6]), it was shown that when the number of acceptable products for the customers increases (i.e., when the preference matrix is less sparse), then the instances are more difficult and take longer to solve. However, due to the amount of 
parameters already considered for this study, we have not included instances with different preference matrix sparsity. Thus, for the instances with $|I|=15$, the number of acceptable products for each customer is equal to 3 , whereas for instances with $|I|=10$ and $|I|=15$ products, the number ascends to 5 acceptable products. In all instances, the number of copies of each product available (called $C$ in the following) is the same for all the products in $I$. So for each combination of the previous parameters, we considered four different sizes for $C$. The reservation prices of the customers were randomly generated between 1 and $4|K|$, and their ranked lists of preferences were also randomly generated. We generated 5 instances of each combination of parameters, 300 in total. All the instances can be found at https://github.com/cdomsa/CRPP/. The time limit was set to 3600 seconds, and the default setting of Xpress was used.

We first compare the performance of MET1-MET7 using the instances of smaller size, namely those with $|K|=50,|K|=75$ and $|K|=100$. The most significant information obtained is summarized by means of several figures. Resolution schemes MET1 and MET3, which consist in solving the instances using formulations (3ICM) and (2ICM) respectively (without valid inequalities), are shown in the legends of the figures as MET1[3ICM] and MET3[2ICM].

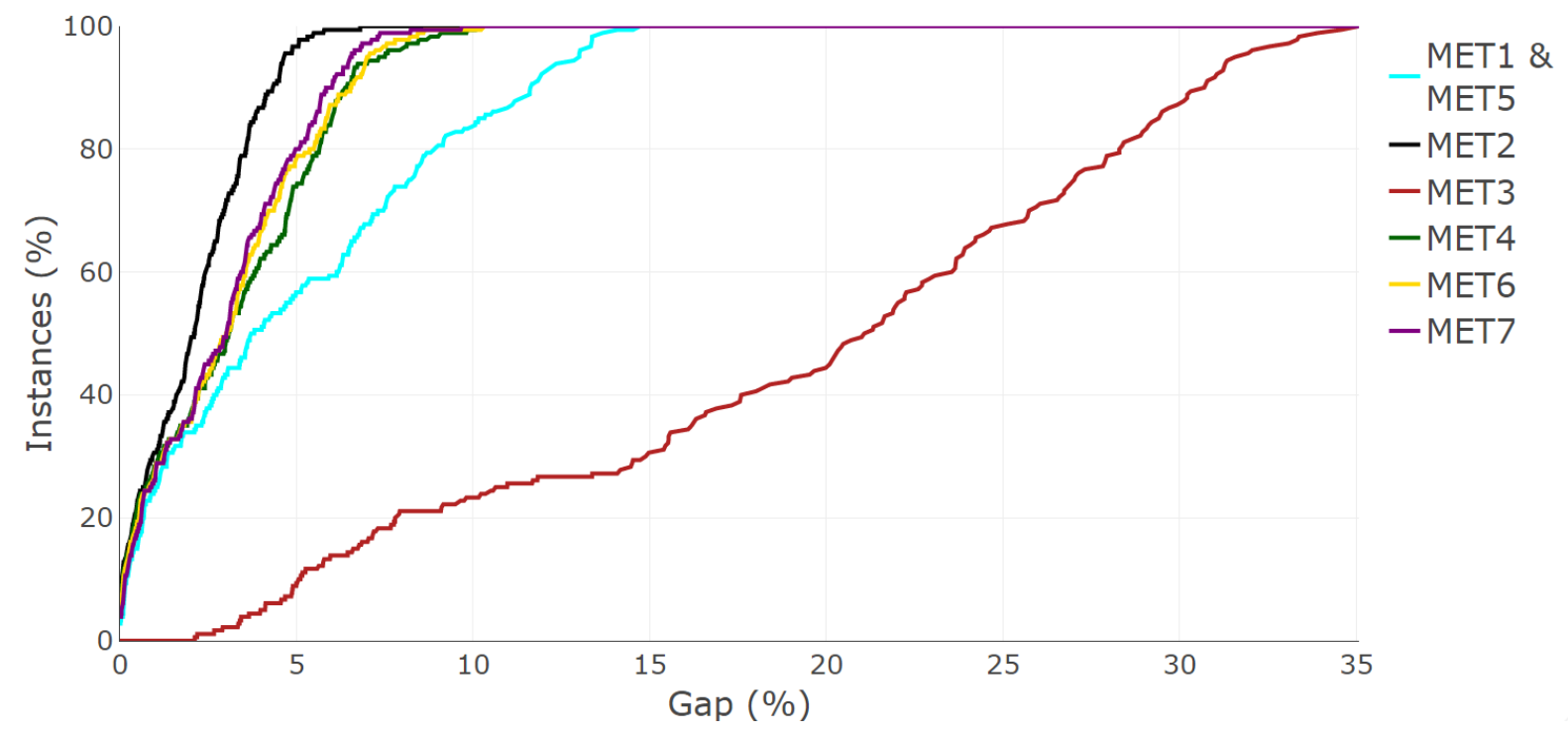

Figure 2: In the $y$-axis, the percentage of instances with an integrality gap less than or equal to that of the $x$-value is represented for MET1-MET7

First, we compare the integrality gaps obtained. Figure 2 is a performance profile that shows the percentage of instances having an integrality gap less than or equal to that on the x-axis. For MET1 and MET3, the gap depicted is the linear relaxation gap LRGap $=100 \frac{\mathrm{UB}-\mathrm{BV}}{\mathrm{BV}} \%$ where UB is the upper bound given by the linear relaxation and $\mathrm{BV}$ is the best objective value found by any of the models (the optimal value almost always). For MET2, MET4-MET7, the figure shows the integrality gap obtained after adding the violated cuts in the linear relaxation, before branching: RGap $=100 \frac{\mathrm{UBC}-\mathrm{BV}}{\mathrm{BV}} \%$, where UBC corresponds to the upper bound obtained after adding the violated cuts in the root node.

As expected, MET3 yields the worst linear relaxation gap of the seven, with gaps of 
up to $35 \%$. MET1 (formulation (3ICM)) has the second worst linear relaxation bound, and this bound is the same for MET5. This is consistent with the results obtained in Section 5. Indeed, valid inequalities (16) are obtained projecting formulation (3ICM) on formulation (2ICM), because the parameters associated to valid inequalities (3), (4) and (9a) are set to 0 in the separation problem. As for MET4, MET6 and MET7, they provide similar integrality gaps, MET7 slightly outperforming the others. These methods yield gaps smaller than $10 \%$ for all the instances proposed. It is remarkable that the gap obtained adding valid inequalities to (2ICM) can outperform that of (3ICM), as it happens in four out of the seven methods developed. Finally, the best scheme in terms of gap is MET2. This is also consistent with our theoretical results, since this method includes a branch-and-cut with all the valid inequalities developed for (3ICM). The gaps provided by MET2 are always smaller than $7 \%$.

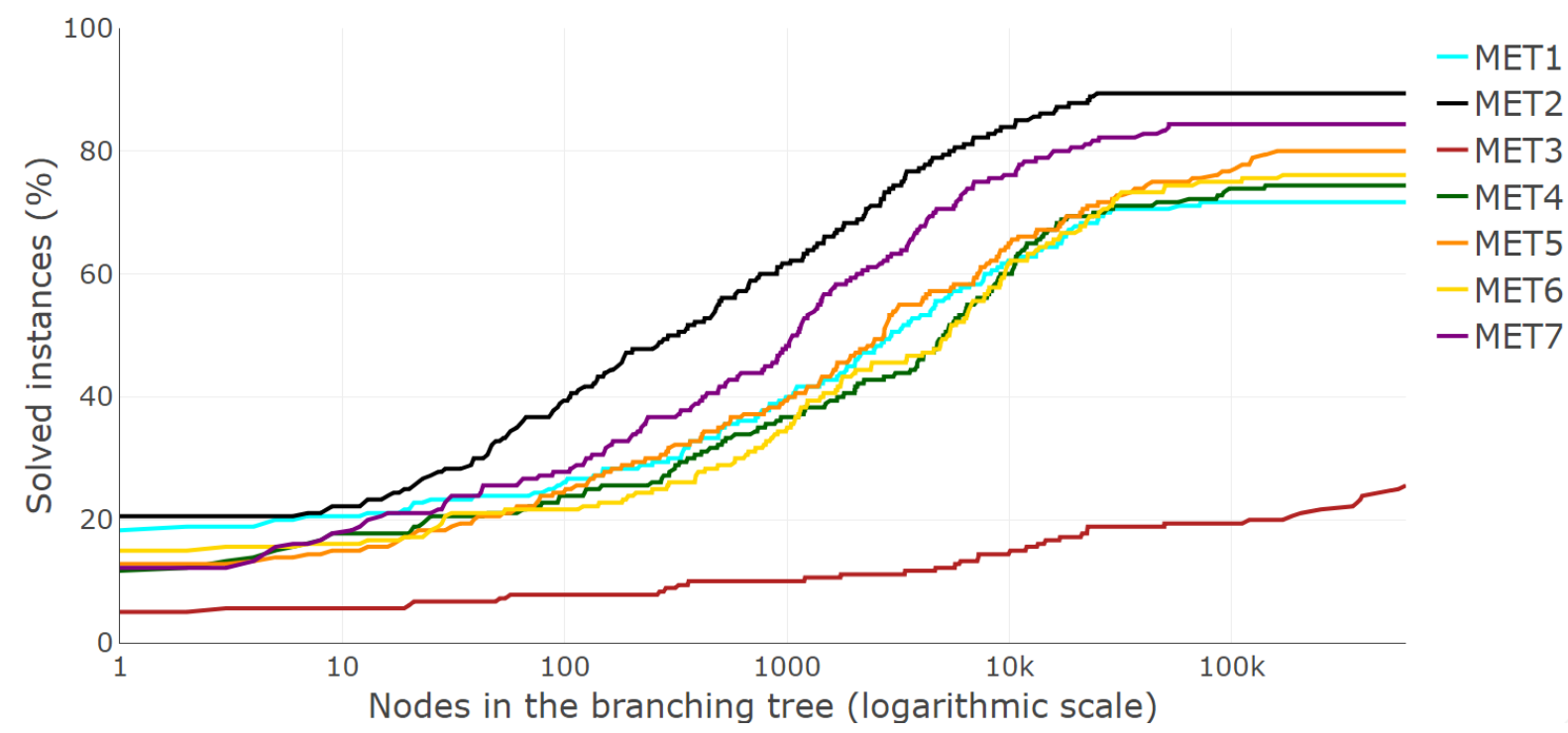

Figure 3: In the $y$-axis, the percentage of instances solved exploring an amount of nodes in the branching tree less than or equal to that of the $x$-value is represented for MET1MET7

Figure 3 compares MET1-MET7 in terms of the number of nodes explored in the branching tree with respect to the percentage of instances solved to optimality. Once again, formulation (2ICM) (MET3) is clearly the worst in performance, it only solves about a $25 \%$ of the instances proposed. Methods MET4-MET6 perform similarly to MET1 (formulation (3ICM)), whereas MET7 performs a bit better than the rest of the approaches, solving up to an $81 \%$ of the instances. Finally, MET2 solves the greatest amount of instances and it does so with the least amount of nodes explored.

Finally, Figure 4 shows the number of instances solved with respect to the time (in seconds), up to 3600 seconds of time limit. This figure confirms the results already seen in the two previous figures, where MET2 and MET3 are the best and worst (respectively) in terms of solved instances. The addition of inequalities to formulation (2ICM) triples the number of instances that are solved to optimality, regardless of the method. Here, the difference in performance between MET4-MET7 is more pronounced than in the previous figures. Thus, we can see that valid inequalities (16) alone (in MET5) perform 


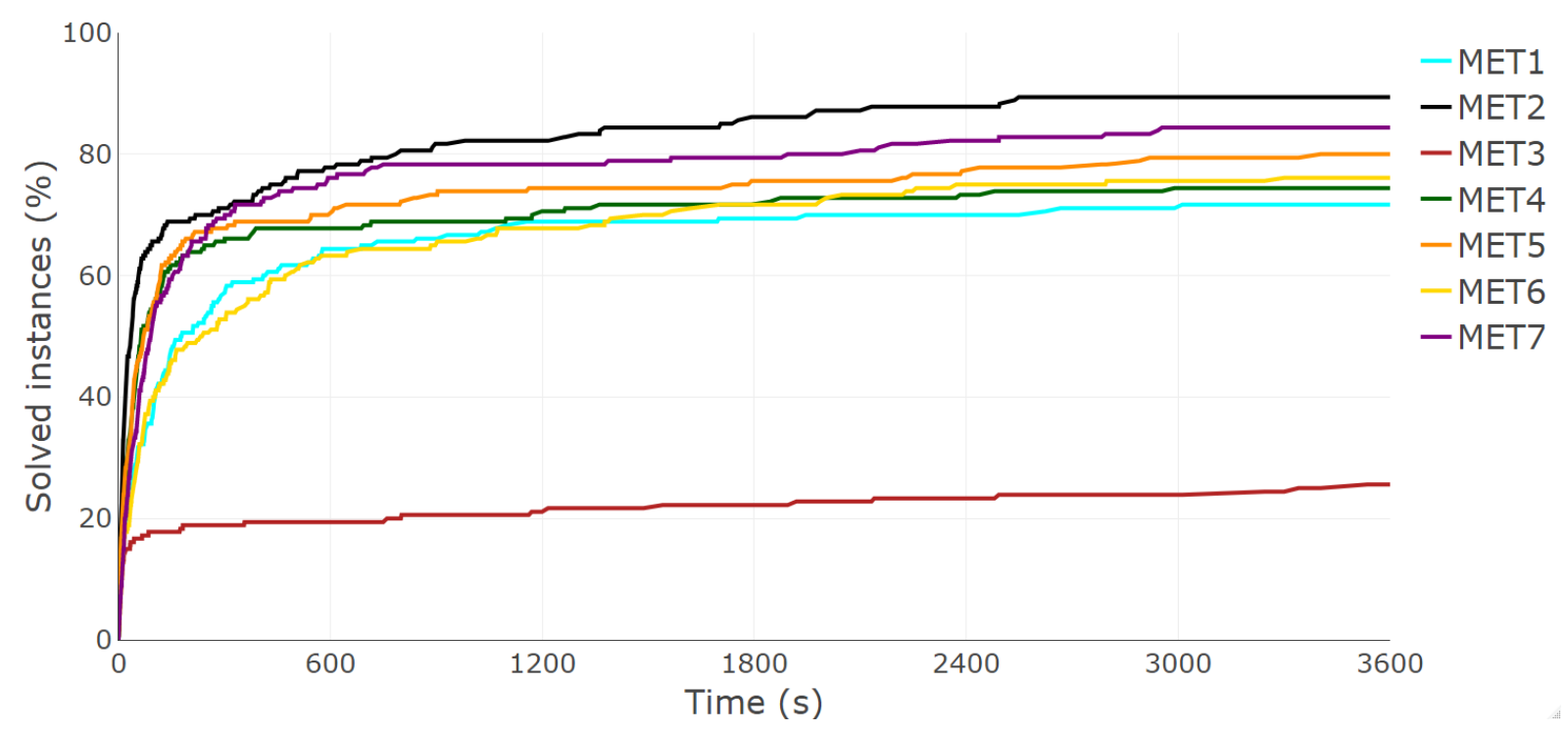

Figure 4: Percentage of instances solved with respect to the time (with a time limit of 3600 seconds) by MET1-MET7

slightly better than (20) (in MET6), even though the upper bound provided by MET6 is smaller. Moreover, the combination of both types of valid inequalities in the branch-andcut makes MET7 outperform the two previous approaches. MET4, the branch-and-cut obtained solving the Farkas separation problem (10), provides the worst results of the group.

In view of the results obtained, we decided to run the largest instances (those with $|K|=125$ and $|K|=150$ ) with the three best methods developed: MET2, MET5 and MET7. Table 2 summarizes the main results obtained. It shows the integrality gap of the linear relaxation (LRGap, the same for MET5 and MET7), the integrality gap of the linear relaxation after the cuts in the root node (RGap), the integrality gap after 3600 seconds (FGap), the average time in seconds needed to optimally solve the instances $(\mathrm{t}(\mathrm{s}))$ and the number of instances solved to optimality in less than the time limit of 3600 seconds. Note that RGap for MET5 is the same as LRGap for MET2.

We can see that the relationship between the number of customers, products and copies of each product determines the number of instances that can be solved to optimality within an hour. In some cases, the three methods can solve all the instances, whereas in other cases none of the instances is solved. Moreover, each method performs better than the rest for certain combinations of parameters. For instance, for a small number of products $(|I|=5)$, MET5 yields the best results in terms of time, even if all the instances are also solved by MET7 within the time limit. However, for $|I|=15$, MET5 is worse than MET7 in terms of time, final bound and number of instances solved. Besides, for greater values of $C$, valid inequalities (20) added in MET7 reduce the gap RGap significantly. For instance, for $|K|=150,|I|=15, C=30$, RGap for MET5 is equal to 14.6, whereas RGap for MET7 is equal to 4.2. As for MET2, it solves less instances than MET5 and MET7. Nonetheless, it provides better final bounds for the combinations of parameters that make the instances hard to solve in one hour. All in all, the inclusion of valid inequalities to models (2ICM) and (3ICM) in a branch-and-cut fashion highly improves their performance. Valid inequalities (16) are essential to reduce the bound of (2ICM) 
regardless of the instance, whereas (20) are particularly useful for instances with a greater number of products and/or copies of each product.

\begin{tabular}{|c|c|c|c|c|c|c|c|c|c|c|c|c|c|c|c|c|}
\hline \multirow{2}{*}{$|K|$} & \multirow{2}{*}{$|I|$} & \multirow{2}{*}{$C$} & \multicolumn{3}{|l|}{ MET2 } & \multicolumn{5}{|c|}{ MET5 } & \multicolumn{6}{|c|}{ MET7 } \\
\hline & & & LRGap & RGap & FGap & $\mathrm{t}(\mathrm{s})$ & Sol. & LRGap & RGap & FGap & $\mathrm{t}(\mathrm{s})$ & Sol. & RGap & FGap & $\mathrm{t}(\mathrm{s})$ & Sol. \\
\hline 125 & 5 & 5 & 0.3 & 0.2 & 0.0 & 10 & 5 & 5.9 & 0.3 & 0.0 & 4 & 5 & 0.3 & 0.0 & 12 & 5 \\
\hline 125 & 5 & 13 & 2.4 & 2.1 & 0.0 & 254 & 5 & 17.8 & 2.4 & 0.0 & 41 & 5 & 2.3 & 0.0 & 65 & 5 \\
\hline 125 & 5 & 20 & 4.0 & 3.6 & 0.0 & 1479 & 5 & 29.8 & 4.0 & 0.0 & 81 & 5 & 3.9 & 0.0 & 196 & 5 \\
\hline 125 & 5 & 25 & 6.2 & 4.5 & 1.0 & 3271 & 2 & 34.7 & 6.2 & 0.0 & 206 & 5 & 5.6 & 0.0 & 520 & 5 \\
\hline 125 & 10 & 5 & 0.7 & 0.6 & 0.0 & 78 & 5 & 8.8 & 0.7 & 0.0 & 53 & 5 & 0.7 & 0.0 & 84 & 5 \\
\hline 125 & 10 & 13 & 4.4 & 3.5 & 3.0 & 3600 & 0 & 23.3 & 4.4 & 1.8 & 3529 & 1 & 4.0 & 2.9 & 3600 & 0 \\
\hline 125 & 10 & 20 & 11.3 & 5.6 & 5.0 & 3600 & 0 & 31.6 & 11.3 & 7.4 & 3600 & 0 & 7.8 & 6.9 & 3600 & 0 \\
\hline 125 & 10 & 25 & 15.2 & 6.7 & 6.3 & 3600 & 0 & 36.6 & 15.2 & 8.9 & 3600 & 0 & 9.0 & 7.5 & 3600 & 0 \\
\hline 125 & 15 & 5 & 1.1 & 0.8 & 0.0 & 266 & 5 & 11.1 & 1.1 & 0.0 & 231 & 5 & 1.0 & 0.0 & 219 & 5 \\
\hline 125 & 15 & 13 & 8.3 & 4.0 & 3.0 & 3101 & 1 & 26.0 & 8.3 & 4.9 & 3600 & 0 & 5.8 & 4.5 & 3600 & 0 \\
\hline 125 & 15 & 20 & 14.2 & 4.7 & 3.7 & 3600 & 0 & 32.6 & 14.2 & 7.7 & 3600 & 0 & 6.4 & 4.6 & 3600 & 0 \\
\hline 125 & 15 & 25 & 15.0 & 4.2 & 2.2 & 3377 & 1 & 33.7 & 15.0 & 7.5 & 3600 & 0 & 4.9 & 1.1 & 2763 & 2 \\
\hline 150 & 5 & 6 & 0.2 & 0.1 & 0.0 & 21 & 5 & 6.5 & 0.2 & 0.0 & 4 & 5 & 0.2 & 0.0 & 18 & 5 \\
\hline 150 & 5 & 15 & 1.8 & 1.5 & 0.0 & 647 & 5 & 16.7 & 1.8 & 0.0 & 57 & 5 & 1.7 & 0.0 & 151 & 5 \\
\hline 150 & 5 & 24 & 4.4 & 4.1 & 2.0 & 3334 & 1 & 30.8 & 4.4 & 0.0 & 127 & 5 & 4.3 & 0.0 & 226 & 5 \\
\hline 150 & 5 & 30 & 7.1 & 4.7 & 4.0 & 3600 & 0 & 35.5 & 7.1 & 0.0 & 395 & 5 & 6.0 & 0.0 & 1259 & 5 \\
\hline 150 & 10 & 6 & 0.8 & 0.7 & 0.0 & 229 & 5 & 9.3 & 0.8 & 0.0 & 177 & 5 & 0.8 & 0.0 & 183 & 5 \\
\hline 150 & 10 & 15 & 4.4 & 3.8 & 3.4 & 3600 & 0 & 24.2 & 4.4 & 3.5 & 3600 & 0 & 4.2 & 3.3 & 3600 & 0 \\
\hline 150 & 10 & 24 & 12.8 & 6.9 & 6.4 & 3600 & 0 & 34.0 & 12.8 & 10.5 & 3600 & 0 & 9.2 & 8.6 & 3600 & 0 \\
\hline 150 & 10 & 30 & 15.7 & 6.9 & 6.6 & 3600 & 0 & 37.2 & 15.7 & 11.9 & 3600 & 0 & 9.5 & 8.7 & 3600 & 0 \\
\hline 150 & 15 & 6 & 1.2 & 0.9 & 0.0 & 1064 & 5 & 12.1 & 1.2 & 0.0 & 1078 & 5 & 1.1 & 0.0 & 683 & 5 \\
\hline 150 & 15 & 15 & 9.2 & 4.9 & 4.4 & 3600 & 0 & 27.8 & 9.2 & 7.3 & 3600 & 0 & 6.8 & 6.2 & 3600 & 0 \\
\hline 150 & 15 & 24 & 13.7 & 4.6 & 4.0 & 3600 & 0 & 33.3 & 13.7 & 10.3 & 3600 & 0 & 6.4 & 4.9 & 3600 & 0 \\
\hline 150 & 15 & 30 & 14.6 & 3.7 & 2.3 & 3421 & 1 & 33.7 & 14.6 & 10.4 & 3600 & 0 & 4.2 & 0.9 & 2318 & 3 \\
\hline
\end{tabular}

Table 2: Results obtained testing MET2, MET5 and MET7 with the instances with 125 and 150 customers ( 5 instances averaged per line). The table includes the integrality gap of the linear relaxation (LRGap), the integrality gap of the linear relaxation after the cuts in the root node (RGap), the integrality gap after 3600 seconds (FGap), the average time in seconds needed to optimally solve the instances $(\mathrm{t}(\mathrm{s}))$ and the number of instances solved to optimality in less than the time limit of 3600 seconds

\section{Conclusions}

In this paper, we studied the CRPP, a generalization of the RPP in which there is a limited number of copies of each product and customers are endowed with different reservation prices for each product of their interest. First, we explained the differences between the allocation of products of the envy-free version of the CRPP and that of the envy version. In this line, we proved that the allocation of products in the CRPP with envy is NPcomplete. Then we presented two mixed-integer formulations along with valid inequalities for each of them. We developed several resolution methods based on the inclusion of valid inequalities in branch-and-cut fashions. To finish, we tested the performance of this methods in terms of integrality gaps, number of nodes and resolution time by means of extensive computational experiments. 
An interesting future line of research consists in modifying the problems' characterization of the customers to make them more realistic and the problem more robust. One way of doing it is considering customers as a combination of different behaviors, where each behavior includes a ranking and the reservation prices associated to a subset of products. In this setting, we could represent the customers' choice rule using a probability mass function over the set of behaviors that would alter the objective function of our models (very much in the spirit of [3]). This approach can also serve as a generalization of the assumption that customers own a fixed reservation price related to each product, and that these reservation prices are known by the company. For instance, we can have descriptions of customers that make choices according to different behaviors which have the same ranking but different reservation prices associated to each product. In this case, the said customers are purchasing according to only one ranking, but they buy at certain prices with certain probabilities.

Another challenging line of research consists in considering the pessimistic approach in the allocation of the products to the customers. In the optimistic case studied, the company can decide on the allocation of a product to the customers to a certain extent (only if it is sold out in the feasible solution). But there are other situations, like considering allocations that depend on the order of arrival of the customers, where the allocation is uncertain and thus it is realistic to assume that the company must optimize the prices for the worst-case scenario.

Finally, a third possible future line of research consists in the study of the sensitivity of the algorithms developed with respect to certain problem parameters, such as the plausible correlation between the customer's reservation prices, the number of acceptable products considered for each customer (related to the sparsity of the instance), et cetera.

\section{Acknowledgements}

The research of Concepción Domínguez has been funded by the Fonds de la Recherche Scientifique - FNRS under a FRIA grant. Concepción Domínguez and Alfredo Marín have been partially supported by the research project MTM2015-65915-R (MINECO, Spain) and by the research project PID2019-110886RB-I00 (MICINN, Spain). Martine Labbé has been partially supported by the Fonds de la Recherche Scientifique -FNRS under Grant PDR T0098.18. The authors thank four anonymous referees for their comments and suggestions that helped improving the paper.

\section{References}

[1] Aggarwal, G., Feder, T., Motwani, R. and Zhu, A. (2004). Algorithms for multiproduct pricing. ICALP, 4, pp. 72-83.

[2] Ben-Ameur, W. and Neto, J. (2007). Acceleration of cutting-plane and column generation algorithms: Applications to network design. Networks: An International Journal, 49(1), 3-17. 
[3] Bertsimas, D. \& Mišić, V.V. (2019). Exact first-choice product line optimization. Operations Research, 67(3), 651-670.

[4] Briest, P. and Krysta, P. (2007). Buying cheap is expensive: Hardness of nonparametric multi-product pricing. In SODA, 7, 716-725.

[5] Calvete, H.I., Domínguez, C., Galé, C., Labbé, M. and Marín, A. (2019). The Rank Pricing Problem: models and branch-and-cut algorithms. Computers $\&$ Operations Research, 105, 12-31.

[6] Domínguez, C., Labbé, M. and Marín, A. (2020). The Rank Pricing Problem with Ties. European Journal of Operational Research, 294(2), 492-506.

[7] Dobson, G. and Kalish, S. (1988). Positioning and pricing a product line. Marketing Science, 7, 107-125.

[8] Dobson, G. and Kalish, S. (1993). Heuristics for pricing and positioning a productline using conjoint and cost data. Management Science, 39, 160-175.

[9] Fernandes, C. G., Ferreira, C. E., Franco, A. J. and Schouery, R. C. (2016). The envy-free pricing problem, unit-demand markets and connections with the network pricing problem. Discrete Optimization, 22, 141-161.

[10] Fischetti, M., Ljubić, I. and Sinnl, M. (2016). Benders decomposition without separability: A computational study for capacitated facility location problems. European Journal of Operational Research, 253(3), 557-569.

[11] Gale, D. and Shapley, L.S. (1962). College admissions and the stability of marriage, American Mathematical Monthly, 69, 9-15.

[12] Guruswami, V., Hartline, J.D., Karlin, A.R., Kempe, D., Kenyon, C. and McSherry, F. (2005). On profit-maximizing envy-free pricing. Proceedings of the sixteenth annual ACM-SIAM symposium on discrete algorithms, 1164-1173.

[13] Heilporn, G., Labbé, M., Marcotte, P. and Savard, G. (2010). A parallel between two classes of pricing problems in transportation and marketing. Journal of Revenue and Pricing Management, 9(1-2), 110-125.

[14] Manlove, D. (2013). Algorithmics of matching under preferences (Vol. 2). World Scientific.

[15] Manlove, D. F., Irving, R.W., Iwama, K., Miyazaki, S. and Morita, Y. (2002). Hard variants of stable marriage. Theoretical Computer Science, 276(1-2), 261-279.

[16] Myklebust, T. G., Sharpe, M. A. and Tunçel, L. (2016). Efficient heuristic algorithms for maximum utility product pricing problems. Computers $\&$ Operations Research, 69, 25-39.

[17] Rusmevichientong, P., Van Roy, B. and Glynn, P.W. (2006). A nonparametric approach to multiproduct pricing. Operations Research, 54(1), 82-98.

[18] Rusmevichientong, P. (2003). A Non-Parametric Approach to Multi-Product Pricing: Theory and Application. Ph.D. thesis. 
[19] Shioda, R., Tunçel, L. and Hui, B. (2014). Applications of deterministic optimization techniques to some probabilistic choice models for product pricing using reservation prices. Pacific Journal of Optimization, 10(4), 767-808.

[20] Shioda, R., Tunçel, L. and Myklebust, T.G. (2011). Maximum utility product pricing models and algorithms based on reservation price. Computational Optimization and Applications, 48(2), 157-198.

[21] Swait, J. (2001). A non-compensatory choice model incorporating attribute cutoffs. Transportation Research Part B: Methodological, 35(10), 903-928.

[22] Van Ryzin, G. and Talluri, K.T. (2004). The Theory and Practice of Revenue Management. Kluwer Academic Publishers. 